(C) 2003 International Press

Adv. Theor. Math. Phys. 7 (2003) 233-268

\title{
Mathematical structure of loop quantum cosmology
}

\author{
Abhay Ashtekar ${ }^{1,2}$, Martin Bojowald ${ }^{1,2}$, and \\ Jerzy Lewandowski ${ }^{3,1,2}$
}

1. Center for Gravitational Physics and Geometry, Physics Department, Penn State, University Park, PA 16802, USA

2. Erwin Schrödinger Institute, Boltzmanngasse 9, 1090 Vienna, Austria

3. Institute of Theoretical Physics, University of Warsaw, ul. Hoża 69, 00-681 Warsaw, Poland

\begin{abstract}
Applications of Riemannian quantum geometry to cosmology have had notable successes. In particular, the fundamental discreteness underlying quantum geometry has led to a natural resolution of the big bang singularity. However, the precise mathematical structure underlying loop quantum cosmology and the sense in which it implements the full quantization program in a symmetry reduced model has not been made explicit. The purpose of this paper is to address these issues, thereby providing a firmer mathematical and conceptual foundation to the subject.
\end{abstract}

\section{Introduction}

In cosmology, one generally freezes all but a finite number of degrees of freedom by imposing spatial homogeneity (and sometimes also isotropy). Because of the resulting mathematical simplifications, the framework provides a simple arena to test ideas and constructions introduced in the full

e-print archive: http://lanl.arXiv.org/abs/gr-qc/0304074 
theory both at the classical and the quantum levels. Moreover, in the classical regime, the symmetry reduction captures the large scale dynamics of the universe as a whole quite well. Therefore, in the quantum theory, it provides a useful test-bed for analyzing the important issues related to the fate of classical singularities.

Over the last three years, ramifications of Riemannian quantum geometry to cosmology have been investigated systematically. First, already at the kinematic level it was found that, thanks to the fundamental discreteness of quantum geometry, the inverse scale factor - and hence also the curvature - remains bounded on the kinematical Hilbert space [1]. Second, while classical dynamics is described by differential equations, the quantum Hamiltonian constraint can be interpreted as providing a difference equation for the 'evolution' of the quantum state [2]. Furthermore, all quantum states remain regular at the classical big-bang; one can 'evolve' right through the point at which classical physics stops [3]. Third, the Hamiltonian constraint together with the requirement - called pre-classicality - that the universe be classical at late times severely restricts the quantum state and, in the simplest models, selects the state uniquely [4]. There are also phenomenological models which allow us to study simple effects of quantum geometry leading to a behavior qualitatively different from the classical one [5]. Finally, the qualitative features are robust [6] and extend also to more complicated cosmological models [7]. These results are quite surprising from the perspective of the 'standard' quantum cosmology which was developed in the framework of geometrodynamics and, together, they show that, once the quantum nature of geometry is appropriately incorporated, the physical predictions change qualitatively in the Planck era.

In spite of these striking advances, the subject has remained incomplete in several respects. First, in the existing treatments, certain subtleties which turn out to have important ramifications were overlooked and the underlying mathematical structure was somewhat oversimplified. This sometimes led to the impression that some of the physically desirable but surprising results arose simply because of ad-hoc assumptions. Second, the essential reasons why loop quantum cosmology is so different from the 'standard' quantum cosmology have not been spelled out. Third, while it is clear that the key constructions and techniques used in loop quantum cosmology are inspired by those developed in the full theory based on quantum geometry $[8,9,10,11,12,13,14,15]$, the parallels and the differences between the full theory and the symmetry reduced models have not been discussed in detail. In this paper, we will address these issues, providing a sounder foundation for the striking results obtained so far. The paper also has a secondary, pedagogical goal: it will also provide an introduction to quantum geome- 
try and loop quantum gravity for readers who are not familiar with these areas. Our discussion should significantly clarify the precise mathematical structure underlying loop quantum cosmology and its relation to the full theory as well as to geometrodynamical quantum cosmology. However, we will not address the most important and the most difficult of open issues: a systematic derivation of loop quantum cosmology from full quantum gravity.

Results of quantum cosmology often provide important qualitative lessons for full quantum gravity. However, while looking for these lessons, it is important to remember that the symmetry reduced theory used here differs from the full theory in conceptually important ways. The most obvious difference is the reduction from a field theory to a mechanical system, which eliminates the potential ultra-violet and infra-red problems of the full theory. In this respect the reduced theory is much simpler. However, there are also two other differences - generally overlooked - which make it conceptually and technically more complicated, at least when one tries to directly apply the techniques developed for the full theory. First, the reduced theory is usually treated by gauge fixing and therefore fails to be diffeomorphism invariant. As a result, key simplifications that occur in the treatment of full quantum dynamics [14] do not carry over. Therefore, in a certain well-defined sense, the non-perturbative dynamics acquires new ambiguities in the reduced theory! The second complication arises from the fact that spatial homogeneity introduces distant correlations. Consequently, at the kinematical level, quantum states defined by holonomies along with distinct edges and triad operators smeared on distinct 2-surfaces are no longer independent. We will see that both these features give rise to certain complications which are not shared by the full theory.

The remainder of this paper is divided in to four sections. In the second, we discuss the phase space of isotropic, homogeneous cosmologies; in the third, we construct the quantum kinematic framework; in the fourth we impose the Hamiltonian constraint and discuss properties of its solutions and in the fifth we summarize the results and discuss some of their ramifications.

\section{Phase space}

For simplicity, we will restrict ourselves to spatially flat, homogeneous, isotropic cosmologies, so that the spatial isometry group $\mathcal{S}$ will be the Euclidean group. Then the 3-dimensional group $T$ of translations (ensuring homogeneity) acts simply and transitively on the 3-manifold $M$. Therefore, $M$ is topologically $R^{3}$. Through the Cartan-Killing form on the Lie-algebra of the 
rotation group, the Lie algebra of translations acquires an equivalence class of positive definite metrics related by an overall constant. Let us fix a metric in this class and an action of the Euclidean group on $M$. This will endow $M$ with a fiducial flat metric ${ }^{\circ} q_{a b}$. Finally, let us fix a constant orthonormal triad ${ }^{o} e_{i}^{a}$ and a co-triad ${ }^{o} \omega_{a}^{i}$ on $M$, compatible with ${ }^{o} q_{a b}$.

Let us now turn to the gravitational phase space in the connection variables. In the full theory, the phase space consists of pairs $\left(A_{a}^{i}, E_{i}^{a}\right)$ of fields on a 3 -manifold $M$, where $A_{a}^{i}$ is an $\mathrm{SU}(2)$ connection and $E_{i}^{a}$ a triplet of vector fields with density weight $1[16]$. (The density weighted orthonormal triad is given by $\gamma E_{i}^{a}$, where $\gamma$ is the Barbero-Immirzi parameter.) Now, a pair $\left(A_{a}^{\prime i}, E_{i}^{\prime a}\right)$ on $M$ will be said to be symmetric if for every $s \in \mathcal{S}$ there exists a local gauge transformation $g: M \rightarrow S U(2)$, such that

$$
\left(s^{*} A^{\prime}, s^{*} E^{\prime}\right)=\left(g^{-1} A^{\prime} g+g^{-1} d g, g^{-1} E^{\prime} g\right) .
$$

As is usual in cosmology, we will fix the local diffeomorphism and gauge freedom. To do so, note first that for every symmetric pair $\left(A^{\prime}, E^{\prime}\right)$ (satisfying the Gauss and diffeomorphism constraints) there exists an unique equivalent pair $(A, E)$ such that

$$
A=\tilde{c}^{o} \omega^{i} \tau_{i}, \quad E=\tilde{p} \sqrt{{ }^{o} q}{ }^{o} e_{i} \tau^{i}
$$

where $\tilde{c}$ and $\tilde{p}$ are constants, carrying the only non-trivial information contained in the pair $\left(A^{\prime}, E^{\prime}\right)$, and the density weight of $E$ has been absorbed in the determinant of the fiducial metric. (Our conventions are such that $\left[\tau_{i}, \tau_{j}\right]=\epsilon_{i j k} \tau^{k}$, i.e., $2 i \tau_{i}=\sigma_{i}$, where $\sigma_{i}$ are the Pauli matrices.)

In terms of $\tilde{p}$, the physical orthonormal triad $e_{i}^{a}$ and its inverse $e_{a}^{i}$ (both of zero density weight) are given by:

$$
e_{i}^{a} \equiv \gamma \tilde{p} \sqrt{\frac{{ }^{o} q}{q}}{ }^{o} e_{i}^{a}=(\operatorname{sgn} \tilde{p})|\gamma \tilde{p}|^{-\frac{1}{2}{ }^{o}} e_{i}^{a}, \quad \text { and } \quad e_{a}^{i}=(\operatorname{sgn} \tilde{p})|\gamma \tilde{p}|^{\frac{1}{2}}{ }^{o} \omega_{a}^{i}
$$

where $q=\operatorname{det} q_{a b}=\left|\operatorname{det} \gamma E_{i}^{a}\right|$, sgn stands for the sign function and $\gamma$ is the Barbero-Immirzi parameter. As in the full theory, the Barbero-Immirzi parameter $\gamma$ and the determinant factors are necessary to convert the (density weighted) momenta $E_{i}^{a}$ in to geometrical (unweighted) triads $e_{i}^{a}$ and cotriads $e_{a}^{i}$. The sign function arises because the connection dynamics phase space contains triads with both orientations and, because we have fixed a fiducial triad ${ }^{o} e_{i}^{a}$, the orientation of the physical triad $e_{i}^{a}$ changes with the sign of $\tilde{p}$. (As in the full theory, we also allow degenerate co-triads which now correspond to $\tilde{p}=0$, for which the triad vanishes.)

Denote by $\mathcal{A}_{S}$ and $\Gamma_{\text {grav }}^{S}$ the subspace of the gravitational configuration space $\mathcal{A}$ and of the gravitational phase space $\Gamma_{\text {grav }}$ defined by (2). Tangent 
vectors $\delta$ to $\Gamma_{\text {grav }}^{S}$ are of the form:

$$
\delta=(\delta A, \delta E), \quad \text { with } \quad \delta A_{a}^{i} \equiv(\delta \tilde{c})^{o} \omega_{a}^{i}, \delta E_{i}^{a} \equiv(\delta \tilde{p})^{o} e_{i}^{a} .
$$

Thus, $\mathcal{A}_{S}$ is 1 -dimensional and $\Gamma_{\text {grav }}^{S}$ is 2-dimensional: we made a restriction to symmetric fields and solved and gauge-fixed the gauge and the diffeomorphism constraints, thereby reducing the local, infinite number of gravitational degrees of freedom to just one.

Because $M$ is non-compact and our fields are spatially homogeneous, various integrals featuring in the Hamiltonian framework of the full theory diverge. This is in particular the case for the symplectic structure of the full theory:

$$
\Omega_{\mathrm{grav}}\left(\delta_{1}, \delta_{2}\right)=\frac{1}{8 \pi \gamma G} \int_{M} d^{3} x\left(\delta_{1} A_{a}^{i}(x) \delta_{2} E_{i}^{a}(x)-\delta_{2} A_{a}^{i}(x) \delta_{1} E_{i}^{a}(x)\right) .
$$

However, the presence of spatial homogeneity enables us to bypass this problem in a natural fashion: Fix a 'cell' $\mathcal{V}$ adapted to the fiducial triad and restrict all integrations to this cell. (For simplicity, we will assume that this cell is cubical with respect to ${ }^{o} q_{a b}$.) Then the gravitational symplectic structure $\Omega_{\text {grav }}$ on $\Gamma_{\text {grav }}$ is given by:

$$
\Omega_{\text {grav }}\left(\delta_{1}, \delta_{2}\right)=\frac{1}{8 \pi \gamma G} \int_{\mathcal{V}} d^{3} x\left(\delta_{1} A_{a}^{i}(x) \delta_{2} E_{i}^{a}(x)-\delta_{2} A_{a}^{i}(x) \delta_{1} E_{i}^{a}(x)\right) .
$$

Using the form (4) of the tangent vectors, the pull-back of $\Omega$ to $\Gamma_{\text {grav }}^{S}$ reduces just to:

$$
\Omega_{\mathrm{grav}}^{S}=\frac{3 V_{o}}{8 \pi \gamma G} d \tilde{c} \wedge d \tilde{p}
$$

where $V_{o}$ is the volume of $\mathcal{V}$ with respect to the auxiliary metric ${ }^{o} q_{a b}$. ( Had $M$ been compact, we could set $\mathcal{V}=M$ and $V_{o}$ would then be the total volume of $M$ with respect to ${ }^{o} q_{a b}$.) Thus, we have specified the gravitational part of the reduced phase space. We will not need to specify matter fields explicitly but only note that, upon similar restriction to symmetric fields and fixing of gauge and diffeomorphism freedom, we are led to a finite dimensional phase space also for matter fields.

In the passage from the full to the reduced theory, we introduced a fiducial metric ${ }^{o} q_{a b}$. There is a freedom in rescaling this metric by a constant: ${ }^{o} q_{a b} \mapsto k^{2 o} q_{a b}$. Under this rescaling the canonical variables $\tilde{c}, \tilde{p}$ transform via $\tilde{c} \mapsto k^{-1} \tilde{c}$ and $\tilde{p} \mapsto k^{-2} \tilde{p}$. (This is analogous to the fact that the scale factor $\tilde{a}=\sqrt{|\tilde{p}|}$ in geometrodynamics rescales by a constant under the change of the fiducial flat metric.) Since rescalings of the fiducial metric do not 
change physics, by themselves $\tilde{c}$ and $\tilde{p}$ do not have direct physical meaning. Therefore, it is convenient to introduce new variables:

$$
c=V_{o}^{\frac{1}{3}} \tilde{c} \quad \text { and } \quad p=V_{o}^{\frac{2}{3}} \tilde{p}
$$

which are independent of the choice of the fiducial metric ${ }^{\circ} q_{a b}$. In terms of these, the symplectic structure is given by

$$
\Omega_{\mathrm{grav}}^{S}=\frac{3}{8 \pi \gamma G} d c \wedge d p
$$

it is now independent of the volume $V_{o}$ of the cell $\mathcal{V}$ and makes no reference to the fiducial metric. In the rest of the paper, we will work with this phase space description. Note that now the configuration variable $c$ is dimensionless while the momentum variable $p$ has dimensions (length) ${ }^{2}$. (While comparing results in the full theory, it is important to bear in mind that these dimensions are different from those of the gravitational connection and the triad there.) In terms of $p$, the physical triad and co-triad are given by:

$$
e_{i}^{a}=(\operatorname{sgn} p)|\gamma p|^{-\frac{1}{2}}\left(V_{o}^{\frac{1}{3} o} e_{i}^{a}\right), \quad \text { and } \quad e_{a}^{i}=(\operatorname{sgn} p)|\gamma p|^{\frac{1}{2}}\left(V_{o}^{-\frac{1}{3} o} \omega_{a}^{i}\right)
$$

Finally, let us turn to constraints. Since the Gauss and the diffeomorphism constraints are already satisfied, there is a single non-trivial Scalar/Hamiltonian constraint (corresponding to a constant lapse):

$$
-\frac{6}{\gamma^{2}} c^{2} \operatorname{sgn} p \sqrt{|p|}+8 \pi G C_{\text {matter }}=0 \text {. }
$$

\section{Quantization: Kinematics}

\subsection{Elementary variables}

Let us begin by singling out 'elementary functions' on the classical phase space which are to have unambiguous quantum analogs. In the full theory, the configuration variables are constructed from holonomies $h_{e}(A)$ associated with edges $e$ and momentum variables, from $E(S, f)$, momenta $E$ smeared with test fields $f$ on 2 -surfaces $[13,17,18,15]$. But now, because of homogeneity and isotropy, we do not need all edges $e$ and surfaces $S$. Symmetric connections $A$ in $\mathcal{A}_{S}$ can be recovered knowing holonomies $h_{e}$ along edges $e$ 
which lie along straight lines in $M$. Similarly, it is now appropriate to smear triads only across squares (with respect to $\left.{ }^{o} q_{a b}\right) .{ }^{1}$

The $\mathrm{SU}(2)$ holonomy along an edge $e$ is given by:

$$
h_{e}(A):=\mathcal{P} \exp \int_{e} A=\cos \frac{\mu c}{2}+2 \sin \frac{\mu c}{2}\left(\dot{e}^{a o} \omega_{a}^{i}\right) \tau^{i}
$$

where $\mu \in(-\infty, \infty)$ (and $\mu V_{o}^{\frac{1}{3}}$ is the oriented length of the edge with respect to $\left.{ }^{\circ} q_{a b}\right)$. Therefore, the algebra generated by sums of products of matrix elements of these holonomies is just the algebra of almost periodic functions of $c$, a typical element of which can be written as:

$$
g(c)=\sum_{j} \xi_{j} e^{i \frac{\mu_{j} c}{2}}
$$

where $j$ runs over a finite number of integers (labelling edges), $\mu_{j} \in R$ and $\xi_{j} \in C$. In the terminology used in the full theory, one can regard a finite number of edges as providing us with a graph (since, because of homogeneity, the edges need not actually meet in vertices now) and the function $g(A)$ as a cylindrical function with respect to that graph. The vector space of these almost periodic functions is, then, the analog of the space Cyl of cylindrical functions on $\mathcal{A}$ in the full theory $[9,10,11,13,18]$. We will call it the space of cylindrical functions of symmetric connections and denote it by $\mathrm{Cyl}_{S}$.

In the full theory, the momentum functions $E(S, f)$ are obtained by smearing the 'electric fields' $E_{i}^{a}$ with an $\operatorname{su}(2)$-valued function $f^{i}$ on a 2 surface $S$. In the homogeneous case, it is natural to use constant test functions $f^{i}$ and let $S$ be squares tangential to the fiducial triad ${ }^{o} e_{i}^{a}$. Then, we have:

$$
E(S, f)=\int_{S} \Sigma_{a b}^{i} f_{i} d x^{a} d x^{b}=p V_{o}^{-\frac{2}{3}} A_{S, f}
$$

where $\Sigma_{a b}^{i}=\eta_{a b c} E^{c i}$ and where $A_{S, f}$ equals the area of $S$ as measured by ${ }^{o} q_{a b}$, times an obvious orientation factor (which depends on $f_{i}$ ). Thus, apart from a kinematic factor determined by the background metric, the momenta are given just by $p$. In terms of classical geometry, $p$ is related to the physical volume of the elementary cell $\mathcal{V}$ via

$$
V=|p|^{\frac{3}{2}} .
$$

\footnotetext{
${ }^{1}$ Indeed, we could just consider edges lying in a single straight line and a single square. We chose not to break the symmetry artificially and consider instead all lines and all squares.
} 
Finally, the only non-vanishing Poisson bracket between these elementary functions is:

$$
\{g(A), p\}=\frac{8 \pi \gamma G}{6} \sum_{j}\left(i \mu_{j} \xi_{j}\right) e^{i \frac{\mu_{j} c}{2}} .
$$

Since the right side is again in $\mathrm{Cyl}_{S}$, the space of elementary variables is closed under the Poisson bracket. Note that, in contrast with the full theory, now the smeared momenta $E(S, f)$ commute with one another since they are all proportional to $p$ because of homogeneity and isotropy. This implies that now the triad representation does exist. In fact it will be convenient to use it later on in this paper.

\subsection{Representation of the algebra of elementary variables}

To construct quantum kinematics, we seek a representation of this algebra of elementary variables. In the full theory, one can use the Gel'fand theory to first find a representation of the $C^{\star}$ algebra Cyl of configuration variables and then represent the momentum operators on the resulting Hilbert space $[8,9,13,18]$. In the symmetry reduced model, we can follow the same procedure. We will briefly discuss the abstract construction and then present the explicit Hilbert space and operators in a way that does not require prior knowledge of the general framework.

Let us begin with the $C^{\star}$ algebra $\mathrm{Cyl}_{S}$ of almost periodic functions on $\mathcal{A}_{S}$ which is topologically $R$. The Gel'fand theory now guarantees that there is a compact Hausdorff space $\bar{R}_{\mathrm{Bohr}}$, the algebra of all continuous functions on which is isomorphic with $\mathrm{Cyl}_{S} . \bar{R}_{\mathrm{Bohr}}$ is called the Bohr compactification of the real line $\mathcal{A}_{S}$, and $\mathcal{A}_{S}$ is densely embedded in it. The Gel'fand theory also implies that the Hilbert space is necessarily $L^{2}\left(\bar{R}_{\text {Bohr }}, d \mu\right)$ with respect to a regular Borel measure $\mu$. Thus, the classical configuration space $\mathcal{A}_{S}$ is now extended to the quantum configuration space $\bar{R}_{\text {Bohr }}$. The extension is entirely analogous to the extension from the space $\mathcal{A}$ of smooth connections to the space $\overline{\mathcal{A}}$ of generalized connections in the full theory $[8,9,13,15]$ and came about because, as in the full theory, our configuration variables are constructed from holonomies. In the terminology used in the full theory, elements $\bar{c}$ of $\bar{R}_{\mathrm{Bohr}}$ are 'generalized symmetric connections'. In the full theory, $\overline{\mathcal{A}}$ is equipped with a natural, faithful, 'induced' Haar measure, which enables one to construct the kinematic Hilbert space and a preferred representation of the algebra of holonomies and smeared momenta $[9,10,11,12,13]{ }^{2}$ Sim-

\footnotetext{
${ }^{2}$ Recently, this representation has been shown to be uniquely singled out by the requirement of diffeomorphism invariance [19, 20, 21].
} 
ilarly, $\bar{R}_{\text {Bohr }}$ is equipped with a natural faithful, 'Haar measure' which we will denote by $\mu_{o}{ }^{3}$

Let us now display all this structure more explicitly. The Hilbert space $\mathcal{H}_{\text {grav }}^{S}=L^{2}\left(\bar{R}_{\mathrm{Bohr}}, d \mu_{o}\right)$ can be made 'concrete' as follows. It is the Cauchy completion of the space $\mathrm{Cyl}_{S}$ of almost periodic functions of $c$ with respect to the inner product:

$$
\left\langle e^{i \frac{\mu_{1} c}{2}} \mid e^{i \frac{\mu_{2} c}{2}}\right\rangle=\delta_{\mu_{1}, \mu_{2}}
$$

(Note that the right side is the Kronecker delta, not the Dirac distribution.) Thus, the almost periodic functions $\mathcal{N}_{\mu}(c):=e^{i \mu c / 2}$ constitute an orthonormal basis in $\mathcal{H}_{\text {grav }}^{S}$. $\mathrm{Cyl}_{S}$ is dense in $\mathcal{H}_{\text {grav }}^{S}$, and serves as a common domain for all elementary operators. The configuration variables act in the obvious fashion: For all $g_{1}$ and $g_{2}$ in $\mathrm{Cyl}_{S}$, we have:

$$
\left(\hat{g}_{1} g_{2}\right)(c)=g_{1}(c) g_{2}(c)
$$

Finally, we represent the momentum operator via

$$
\hat{p}=-i \frac{\gamma \ell_{\mathrm{Pl}}^{2}}{3} \frac{d}{d c}, \quad \text { whence, } \quad(\hat{p} g)(c)=\frac{\gamma \ell_{\mathrm{Pl}}^{2}}{6} \sum_{j}\left[\xi_{j} \mu_{j}\right] \mathcal{N}_{\mu_{j}}
$$

where $g \in \mathrm{Cyl}_{S}$ is given by (13) and, following conventions of loop quantum cosmology, we have set $\ell_{\mathrm{Pl}}^{2}=8 \pi G \hbar$. (Unfortunately, this convention is different from that used in much of quantum geometry where $G \hbar$ is set equal to $\ell_{\mathrm{Pl}}$.)

As in the full theory, the configuration operators are bounded, whence their action can be extended to the full Hilbert space $\mathcal{H}_{\text {grav }}^{S}$, while the momentum operators are unbounded but essentially self-adjoint. The basis vectors $\mathcal{N}_{\mu}$ are normalized eigenstates of $\hat{p}$. As in quantum mechanics, let us use the bra-ket notation and write $\mathcal{N}_{\mu}(c)=\langle c \mid \mu\rangle$. Then,

$$
\hat{p}|\mu\rangle=\frac{\mu \gamma \ell_{\mathrm{Pl}}^{2}}{6}|\mu\rangle \equiv p_{\mu}|\mu\rangle .
$$

\footnotetext{
${ }^{3} \bar{R}_{\text {Bohr }}$ is a compact Abelian group and $d \mu_{o}$ is the Haar measure on it. In non-relativistic quantum mechanics, using $\bar{R}_{\text {Bohr }}$ one can introduce a new representation of the standard Weyl algebra. It is inequivalent to the standard Schrödinger representation and naturally incorporates the idea that spatial geometry is discrete at a fundamental scale. Nonetheless, it reproduces the predictions of standard Schrödinger quantum mechanics within its domain of validity. (For details, see [22]). There is a close parallel with the situation in quantum cosmology, where the role of the Schrödinger representation is played by 'standard' quantum cosmology of geometrodynamics.
} 
Using the relation $V=|p|^{3 / 2}$ between $p$ and physical volume of the cell $\mathcal{V}$ we have:

$$
\hat{V}|\mu\rangle=\left(\frac{\gamma|\mu|}{6}\right)^{\frac{3}{2}} \ell_{\mathrm{Pl}}^{3}|\mu\rangle \equiv V_{\mu}|\mu\rangle .
$$

This provides us with a physical meaning of $\mu$ : apart from a fixed constant, $|\mu|^{3 / 2}$ is the physical volume of the cell $\mathcal{V}$ in Planck units, when the universe is in the quantum state $|\mu\rangle$. Thus, in particular, while the volume $V_{o}$ of the cell $\mathcal{V}$ with respect to the fiducial metric ${ }^{\circ} q_{a b}$ may be 'large', its physical volume in the quantum state $|\mu=1\rangle$ is $(\gamma / 6)^{3 / 2} \ell_{\mathrm{Pl}}^{3}$. This fact will be important in sections 3.3 and 4.1 .

Note that the construction of the Hilbert space and the representation of the algebra is entirely parallel to that in the full theory. In particular, $\mathrm{Cyl}_{S}$ is analogous to $\mathrm{Cyl} ; \bar{R}_{\mathrm{Bohr}}$ is analogous to $\overline{\mathcal{A}} ; \mathcal{N}_{\mu}$ to the spin network states $\mathcal{N}_{\alpha, \mathbf{j}, \mathbf{I}}$ (labelled by a graph $g$ whose edges are assigned half integers $\mathbf{j}$ and whose vertices are assigned intertwiners $\mathbf{I}[23,24,18]) . \hat{g}$ are the analogs of configuration operators defined by elements of $\mathrm{Cyl}$ and $\hat{p}$ is analogous to the triad operators. In the full theory, holonomy operators are well-defined but there is no operator representing the connection itself. Similarly, $\hat{\mathcal{N}}_{\mu}$ are well defined unitary operators on $\mathcal{H}_{\text {grav }}^{S}$ but they fail to be continuous with respect to $\mu$, whence there is no operator corresponding to $c$ on $\mathcal{H}_{\text {grav }}^{S}$. Thus, to obtain operators corresponding to functions on the gravitational phase space $\Gamma_{\text {grav }}^{S}$ we have to first express them in terms of our elementary variables $\mathcal{N}_{\mu}$ and $p$ and then promote those expressions to the quantum theory. Again, this is precisely the analog of the procedure followed in the full theory.

There is, however, one important difference between the full and the reduced theories: while eigenvalues of the momentum (and other geometric) operators in the full theory span only a discrete subset of the real line, now every real number is a permissible eigenvalue of $\hat{p}$. This difference can be directly attributed to the high degree of symmetry. In the full theory, eigenvectors are labelled by a pair $(e, j)$ consisting of continuous label $e$ (denoting an edge) and a discrete label $j$ (denoting the 'spin' on that edge), and the eigenvalue is dictated by $j$. Because of homogeneity and isotropy, the pair $(e, j)$ has now collapsed to a single continuous label $\mu$. Note however that there is a weaker sense in which the spectrum is discrete: all eigenvectors are normalizable. Hence the Hilbert space can be expanded out as a direct sum - rather than a direct integral - of the 1-dimensional eigenspaces of $\hat{p}$; i.e., the decomposition of identity on $\mathcal{H}_{S}$ is given by a (continuous) sum

$$
I=\sum_{\mu}|\mu\rangle\langle\mu|
$$


rather than an integral. Although weaker, this discreteness is nonetheless important both technically and conceptually. In the next sub-section, we present a key illustration.

We will conclude with two remarks.

i) In the above discussion we worked with $c, p$ rather than the original variables $\tilde{c}, \tilde{p}$ to bring out the physical meaning of various objects more directly. Had we used the tilde variables, our symplectic structure would have involved $V_{o}$ and we would have had to fix $V_{o}$ prior to quantization. The Hilbert space and the representation of the configuration operators would have been the same for all choices of $V_{o}$. However, the representation of the momentum operators would have changed from one $V_{o}$ sector to another: A change $V_{o} \mapsto k^{3} V_{o}$ would have implied $\hat{p} \mapsto k^{-2} \hat{p}$. The analogous transformation is not unitarily implementable in Schrödinger quantum mechanics nor in full quantum gravity. However, somewhat surprisingly, it is unitarily implementable in the reduced model. ${ }^{4}$ Therefore, quantum physics does not change with the change of $V_{o}$. Via untilded variables, we chose to work with an unitarily equivalent representation which does not refer to $V_{o}$ at all.

ii) For simplicity of presentation, in the above discussion we avoided details of the Bohr compactification and worked with its dense space $\mathrm{Cyl}_{S}$ instead. In terms of the compactification, the situation can be summarized as follows. After Cauchy completion, each element of $\mathcal{H}_{\text {grav }}^{S}$ is represented by a square-integrable function $f(\bar{c})$ of generalized symmetric connections. By Gel'fand transform, every element $g$ of $\mathrm{Cyl}_{S}$ is represented by a function $\check{g}(\bar{c})$ on $\mathrm{Cyl}_{S}$ and the configuration operators act via multiplication on the full Hilbert space: $\left(\hat{g}_{1} g_{2}\right)(\bar{c})=\check{g}_{1}(\bar{c}) g_{2}(\bar{c})$. The momentum operator $\hat{p}$ is essentially self-adjoint on the domain consisting of the image of $\mathrm{Cyl}_{S}$ under the Gel'fand transform.

\subsection{Triad operator}

In the reduced classical theory, curvature is simply a multiple of the inverse of the square of the scale factor $a=\sqrt{|p|}$. Similarly, the matter Hamiltonian invariably involves a term corresponding to an inverse power of $a$. Therefore,

\footnotetext{
${ }^{4}$ The difference from Schrödinger quantum mechanics can be traced back to the fact that the eigenvectors $|p\rangle$ of the Schrödinger momentum operator satisfy $\left\langle p \mid p^{\prime}\right\rangle=\delta\left(p, p^{\prime}\right)$ while the eigenvectors of our $\hat{p}$ in the reduced model satisfy $\left\langle\mu \mid \mu^{\prime}\right\rangle=\delta_{\mu, \mu^{\prime}}$, the Dirac delta distribution being replaced by the Kronecker delta. In full quantum gravity, one also has the Kronecker-delta normalization for the eigenvectors of triad (and other geometrical) operators. Now the difference arises because there the eigenvalues form a discrete subset of the real line while in the symmetry reduced model they span the full line.
} 
we need to obtain an operator corresponding to the inverse scale factor, or the triad (with density weight zero) of (10). In the classical theory, the triad coefficient diverges at the big bang and a key question is whether quantum effects 'tame' the big bang sufficiently to make the triad operator (and hence the curvature and the matter Hamiltonian) well behaved there.

Now, in non-relativistic quantum mechanics, the spectrum of the operator $\hat{r}$ is the positive half of the real line, equipped with the standard Lesbegue measure, whence the operator $1 / \hat{r}$ is a densely-defined, self-adjoint operator. By contrast, since $\hat{p}$ admits a normalized eigenvector $|\mu=0\rangle$ with zero eigenvalue, the naive expression of the triad operator fails to be densely defined on $\mathcal{H}_{\text {grav }}^{S}$. One could circumvent this problem in the reduced model in an ad-hoc manner by just making up a definition for the action of the triad operator on $|\mu=0\rangle$. But then the result would have to be considered as an artifact of a procedure expressly invented for the model and one would not have any confidence in its implications for the big bang. Now, as one might expect, a similar problem arises also in the full theory. There, a mathematically successful strategy to define the required operators already exits [14]: One first re-expresses the desired, potentially 'problematic' phase space function as a regular function of elementary variables and the volume function and then replaces these by their well-defined quantum analogs. It is appropriate to use the same procedure also in quantum cosmology; not only is this a natural approach but it would also test the general strategy. As in the general theory, therefore, we will proceed in two steps. In the first, we note that, on the reduced phase space $\Gamma_{\text {grav }}^{S}$, the triad coefficient $\operatorname{sgn} p|p|^{-\frac{1}{2}}$ can be expressed as the Poisson bracket $\left\{c, V^{1 / 3}\right\}$ which can be replaced by $i \hbar$ times the commutator in quantum theory. However, a second step is necessary because there is no operator $\hat{c}$ on $\mathcal{H}_{\text {grav }}^{S}$ corresponding to $c$ : one has to re-express the Poisson bracket in terms of holonomies which do have unambiguous quantum analogs.

Indeed, on $\Gamma_{\text {grav }}^{S}$, we have:

$$
\frac{\operatorname{sgn}(p)}{\sqrt{|p|}}=\frac{4}{8 \pi G \gamma} \operatorname{tr}\left(\sum_{i} \tau^{i} h_{i}\left\{h_{i}^{-1}, V^{\frac{1}{3}}\right\}\right),
$$

where

$$
h_{i}:=\mathcal{P} \exp \left(\stackrel{V_{0}^{\frac{1}{3}}}{\int_{0} o} e_{i}^{a} A_{a}^{j} \tau_{j} d t\right)=\exp \left(c \tau_{i}\right)=\cos \frac{c}{2}+2 \sin \frac{c}{2} \tau_{i}
$$

is the holonomy (of the connection $A_{a}^{i}$ ) evaluated along an edge along the elementary cell $\mathcal{V}$ (i.e., an edge parallel to the triad vector ${ }^{o} e_{i}^{a}$ of length $V_{o}^{1 / 3}$ 
with respect to the fiducial metric ${ }^{o} q_{a b}$ ), and where we have summed over $i$ to avoid singling out a specific triad vector. ${ }^{5}$ We can now pass to quantum theory by replacing the Poisson brackets by commutators. This yields the triad (coefficient) operator:

$$
\begin{aligned}
{\left[\widehat{\left.\frac{\operatorname{sgn}(p)}{\sqrt{|p|}}\right]}\right.} & =-\frac{4 i}{\gamma \ell_{\mathrm{Pl}}^{2}} \operatorname{tr}\left(\sum_{i} \tau^{i} \hat{h}_{i}\left[\hat{h}_{i}^{-1}, \hat{V}^{\frac{1}{3}}\right]\right) \\
& =-\frac{12 i}{\gamma \ell_{\mathrm{Pl}}^{2}}\left(\sin \frac{c}{2} \hat{V}^{\frac{1}{3}} \cos \frac{c}{2}-\cos \frac{c}{2} \hat{V}^{\frac{1}{3}} \sin \frac{c}{2}\right)
\end{aligned}
$$

Although this operator involves both configuration and momentum operators, it commutes with $\hat{p}$, whence its eigenvectors are again $|\mu\rangle$. The eigenvalues are given by:

$$
\left[\widehat{\frac{\operatorname{sgn}(p)}{\sqrt{|p|}}}\right]|\mu\rangle=\frac{6}{\gamma \ell_{\mathrm{Pl}}^{2}}\left(V_{\mu+1}^{1 / 3}-V_{\mu-1}^{1 / 3}\right)|\mu\rangle .
$$

where $V_{\mu}$ is the eigenvalue of the volume operator (see (21)). Next, we note a key property of the triad operator: It is bounded above! The upper bound is obtained at the value $\mu=1$ :

$$
|p|_{\max }^{-\frac{1}{2}}=\sqrt{\frac{12}{\gamma}} \ell_{\mathrm{Pl}}^{-1} .
$$

This is a striking result because $p$ admits a normalized eigenvector with zero eigenvalue. Since in the classical theory the curvature is proportional to $p^{-1}$, in quantum theory, it is bounded above by $(12 / \gamma) \ell_{\mathrm{Pl}}^{-2}$. Note that $\hbar$ is essential for the existence of this upper bound; as $\hbar$ tends to zero, the bound goes to infinity just as one would expect from classical considerations. This is rather reminiscent of the situation with the ground state energy of the hydrogen atom in non-relativistic quantum mechanics, $E_{o}=-\left(m_{\mathrm{e}} e^{4} / 2\right)(1 / \hbar)$, which is bounded from below because $\hbar$ is non-zero.

In light of this surprising result, let us re-examine the physical meaning of the quantization procedure. (Using homogeneity and isotropy, we can naturally convert volume scales in to length scales. From now on, we will do so freely.) In the classical Poisson bracket, we replaced the connection coefficient $c$ by the holonomy along an edge of the elementary cell $\mathcal{V}$ because there is no operator on $\mathcal{H}_{\text {grav }}^{S}$ corresponding to $c$. Since the cell has volume $V_{o}$ with respect to the fiducial metric ${ }^{o} q_{a b}$, the edge has length $V_{o}^{1 / 3}$. While

\footnotetext{
${ }^{5}$ Note that, because of the factors $h_{i}$ and $h_{i}^{-1}$ in this expression, the length of the edge is actually irrelevant. For further discussion, see the remark at the end of this sub-section.
} 
this length can be large, what is relevant is the physical length of this edge and we will now present two arguments showing that the physical length is of the order of a Planck length. The first uses states. Let us begin by noting that, being a function of the connection, (matrix elements of) the holonomy itself determine a quantum state $\mathcal{N}_{\mu=1}(A)=e^{i \frac{c}{2}}$. In this state, the physical volume of the cell $\mathcal{V}$ is not $V_{o}$ but $(\gamma / 6)^{3 / 2} \ell_{\mathrm{Pl}}^{3}$. Hence, the appropriate physical edge length is $(\gamma / 6)^{1 / 2} \ell_{\mathrm{Pl}}$, and this is only of the order of the Planck length. ${ }^{6}$ The same conclusion is reached by a second argument based on the operator $\hat{h}_{i}$ : Since $e^{i \frac{c}{2}}|\mu\rangle=|\mu+1\rangle$ for any $\mu$, the holonomy operator changes the volume of the universe by 'attaching' edges of physical length $(\gamma / 6)^{1 / 2}\left(|\mu+1|^{1 / 2}-|\mu|^{1 / 2}\right) \ell_{\mathrm{Pl}} \cdot{ }^{7}$ These arguments enable us to interpret the quantization procedure as follows. There is no direct operator analog of $c$; only holonomy operators are well-defined. The 'fundamental' triad operator (25) involves holonomies along Planck scale edges. In the classical limit, we can let the edge length go to zero and then this operator reduces to the classical triad, the Poisson bracket $\left\{c, V^{1 / 3}\right\}$.

Since the classical triad diverges at the big bang, it is perhaps not surprising that the 'regularization' introduced by quantum effects ushers-in the Planck scale. However, the mechanism by which this came about is new and conceptually important. For, we did not introduce a cut-off or a regulator; the classical expression (23) of the triad coefficient we began with is exact. Since we did not 'regulate' the classical expression, the issue of removing the regulator does not arise. Nonetheless, it is true that the quantization procedure is 'indirect'. However, this was necessary because the spectrum of the momentum operator $\hat{p}$ (or of the 'scale factor operator' corresponding to $a$ ) is discrete in the sense detailed in section 3.2. Had the Hilbert space $\mathcal{H}_{\text {grav }}^{S}$ been a direct integral of the eigenspaces of $\hat{p}$-rather than a direct sum - the triad operator could then have been defined directly using the spectral decomposition of $\hat{p}$ and would have been unbounded above.

Indeed, this is precisely what happens in geometrodynamics. There, $p$ and $c$ themselves are elementary variables and the Hilbert space is taken to be the space of square integrable functions of $p$ (or, rather, of $a \sim|p|^{1 / 2}$ ). Then, $p$ has a genuinely continuous spectrum and its inverse is a self-adjoint operator, defined in terms the spectral decomposition of $p$ and is unbounded above. By contrast, in loop quantum gravity, quantization is based on holonomies - the Wilson lines of the gravitational connection. We carried this central idea to the symmetry reduced model. As a direct result, as in the full theory,

\footnotetext{
${ }^{6}$ Black hole entropy calculations imply that we should set $\gamma=\frac{\ln 2}{\sqrt{3} \pi}$ to recover the standard quantum field theory in curved space-times from quantum geometry. [26].

${ }^{7}$ The square-root of $\mu$ features rather than $\mu$ itself because $\hat{p}$ corresponds to the square of the scale factor $a$ and we chose to denote its eigenvalues by $(\gamma \mu / 6) \ell_{\mathrm{Pl}}^{2}$.
} 
we were led to a non-standard Hilbert space $\mathcal{H}_{\text {grav }}^{S}$. Furthermore, we were led to consider almost periodic functions of $c$-rather than $c$ itself- as 'elementary' and an operator corresponding to $c$ is not even defined on $\mathcal{H}_{\text {grav }}^{S}$. All eigenvectors of $p$ are now normalizable, including the one with zero eigenvalue. Hence, to define the triad operator, one simply can not repeat the procedure of geometrodynamics. We are led to use the alternate procedure followed above. Of course one could simply invent a regularization scheme just for this symmetry reduced model. A key feature of our procedure is that it was not so invented; it is the direct analog of the procedure followed to address the same issue in the full theory [14].

Finally, let us return to the expression of the quantum operator (25). Since the fact that it is bounded is surprising, it is important to verify that the final result has physically reasonable properties. The first obvious requirement is that, since the triad coefficient $\operatorname{sgn} p /|p|^{\frac{1}{2}}$ is a function only of $p$, the triad operator should commute with $\hat{p}$. A priori there is no guarantee that this would be the case. Indeed, the expression (25) of the triad operator involves $c$ as well. However, as we saw, this condition is in fact met. A second non-trivial requirement comes from the fact that the triad coefficient and the momentum are algebraically related in the classical theory: $p \cdot\left(\operatorname{sgn} p /|p|^{1 / 2}\right)^{2}=1$. A key criterion of viability of the triad operator is that this relation should be respected in an appropriate sense. More precisely, we can tolerate violations of this condition on states only in the Planck regime; the equality must be satisfied to an excellent approximation on states with large $\mu$ (i.e., with large volume). Is this the case? We have:

$$
\begin{aligned}
\frac{6}{\gamma \ell_{\mathrm{Pl}}^{2}}\left(V_{\mu+1}^{1 / 3}-V_{\mu-1}^{1 / 3}\right) & =\sqrt{\frac{6|\mu|}{\gamma \ell_{\mathrm{Pl}}^{2}}}(\sqrt{|1+1 / \mu|}-\sqrt{|1-1 / \mu|}) \\
& =\operatorname{sgn} \mu \sqrt{\frac{6}{\gamma|\mu| \ell_{\mathrm{Pl}}^{2}}}\left(1+O\left(\mu^{-2}\right)\right)
\end{aligned}
$$

Thus, up to order $O\left(\mu^{-2}\right)$, the eigenvalue of the triad operator is precisely $\operatorname{sgn} p_{\mu} / \sqrt{\left|p_{\mu}\right|}$, where $p_{\mu}$ is the eigenvalue of $\hat{p}$ (see $(21)$ ). On states representing a large universe $(|\mu| \gg 1)$, the classical algebraic relation between the triad coefficient and $p$ is indeed preserved to an excellent approximation. Violations are significant only on the eigen-subspace of the volume operator with eigenvalues of the order of $\ell_{\mathrm{Pl}}^{3}$ or less, i.e., in the fully quantum regime.

Remark: In (23), we used holonomies along edges of the elementary cell $\mathcal{V}$. While this choice is natural because the cell is needed for classical considerations in any case, one might imagine using, instead, edges of length $\left|\mu_{o}\right| V_{o}^{1 / 3}$. Had this been done, the replacement of (23) would have again 
provided an exact expression of the triad coefficient in the classical theory. However, to meet the second criterion above, one would be forced to choose $\left|\mu_{o}\right| \sim 1$ and we would be back with the 'natural' choice made above. Nonetheless, since there is a quantization ambiguity, the numerical coefficients in the final results (e.g., the precise value of the upper bound of the triad operator spectrum) should not be attached direct physical significance. In particular, for lessons for the full theory, one should use only the qualitative features and orders of magnitudes. The numerical values can be arrived at only through a systematic reduction of the full quantum theory, where the precise value of $\left|\mu_{o}\right|(\sim 1)$ should emerge, e.g., as the lowest eigenvalue of an appropriate geometric operator. We will return to this issue in the next section.

\section{Quantum dynamics: The Hamiltonian constraint}

Since the curvature is bounded above on the entire kinematical Hilbert space $\mathcal{H}_{\text {grav }}^{S}$, one might expect that the classical singularity at the big bang would be naturally resolved in the quantum theory. In this section we will show that this is indeed the case.

\subsection{The quantum Hamiltonian constraint}

In section 1, we reduced the Hamiltonian constraint to (11). However, we can not use this form of the constraint directly because it is cast in terms of the connection $c$ itself rather than holonomies. One can 'regulate' it in terms of holonomies and then pass to quantum theory. However, to bring out the close similarity of the regularization procedure with the one followed in the full theory [14], we will obtain the same expression starting from the expression of the classical constraint in the full theory:

$$
\begin{aligned}
C_{\text {grav }} & :=\int_{\mathcal{V}} d^{3} x N e^{-1}\left(\epsilon_{i j k} F_{a b}^{i} E^{a j} E^{b k}-2\left(1+\gamma^{2}\right) K_{[a}^{i} K_{b]}^{j} E_{i}^{a} E_{j}^{b}\right) \\
& =-\gamma^{-2} \int_{\mathcal{V}} d^{3} x N \epsilon_{i j k} F_{a b}^{i} e^{-1} E^{a j} E^{b k}
\end{aligned}
$$

where $e:=\sqrt{|\operatorname{det} E|} \operatorname{sgn}(\operatorname{det} E)$. We restricted the integral to our cell $\mathcal{V}$ (of volume $V_{o}$ with respect to ${ }^{\circ} q_{a b}$ ) and, in the second step, exploited the fact that for spatially flat, homogeneous models the two terms in the full constraint are proportional to each other (one can also treat both terms as in the full theory without significant changes [25]). Because of homogeneity, 
we can assume that the lapse $N$ is constant and, for definiteness, from now onwards we will set it to one.

As a first step in constructing a Hamiltonian constraint operator we have to express the curvature components $F_{a b}^{i}$ in terms of holonomies. We will use the procedure followed in the full theory [14] (or in lattice gauge theories). Consider a square $\alpha_{i j}$ in the $i-j$ plane spanned by two of the triad vectors ${ }^{o} e_{i}^{a}$, each of whose sides has length $\mu_{o} V_{o}^{1 / 3}$ with respect to the fiducial metric ${ }^{o} q_{a b} .{ }^{8}$ Then, 'the $a b$ component' of the curvature is given by

$$
F_{a b}^{i} \tau_{i}={ }^{o} \omega_{a}^{i}{ }^{o} \omega_{b}^{j}\left(\frac{h_{\alpha_{i j}}^{\left(\mu_{o}\right)}-1}{\mu_{o}^{2} V_{o}^{2 / 3}}+O\left(c^{3} \mu_{o}\right)\right)
$$

The holonomy $h_{\alpha_{i j}}^{\left(\mu_{o}\right)}$ in turn can be expressed as

$$
h_{\alpha_{i j}}^{\left(\mu_{o}\right)}=h_{i}^{\left(\mu_{o}\right)} h_{j}^{\left(\mu_{o}\right)}\left(h_{i}^{\left(\mu_{o}\right)}\right)^{-1}\left(h_{j}^{\left(\mu_{o}\right)}\right)^{-1}
$$

where, as before, holonomies along individual edges are given by

$$
h_{i}^{\left(\mu_{o}\right)}:=\cos \frac{\mu_{o} c}{2}+2 \sin \frac{\mu_{o} c}{2} \tau_{i}
$$

Next, let us consider the triad term $\epsilon_{i j k} e^{-1} E^{a j} E^{b k}$ in the expression of the Hamiltonian constraint. Since the triad is allowed to become degenerate, there is a potential problem with the factor $e^{-1}$. In the reduced model, $e$ vanishes only when the triad itself vanishes and hence the required term $\epsilon_{i j k} e^{-1} E^{a j} E^{b k}$ can be expressed as a non-singular function of $p$ and the fiducial triads. In the full theory, the situation is more complicated and such a direct approach is not available. There is nonetheless a procedure to handle this apparently singular function [14]: one expresses it as a Poisson bracket between holonomies and the volume function as in section 3.3 and then promotes the resulting expression to an operator. To gain insight in to this strategy, here we will follow the same procedure. Thus, let us begin with the identity on the symmetry reduced phase space $\Gamma_{\text {grav }}^{S}$ :

$$
\epsilon_{i j k} \tau^{i} e^{-1} E^{a j} E^{b k}=-2\left(8 \pi \gamma G \mu_{o} V_{o}^{1 / 3}\right)^{-1} \epsilon^{a b c o} \omega_{c}^{k} h_{k}^{\left(\mu_{o}\right)}\left\{h_{k}^{\left(\mu_{o}\right)-1}, V\right\}
$$

where $h_{k}^{\left(\mu_{0}\right)}$ is the holonomy along the edge parallel to the $k$ th basis vector of length $\mu_{o} V_{o}^{1 / 3}$ with respect to ${ }^{o} q_{a b}$. Note that, unlike the expression (30) for $F_{a b}^{i},(33)$ is exact, i.e. does not depend on the choice of $\mu_{o}$.

\footnotetext{
${ }^{8}$ In a model with non-zero intrinsic curvature, those edges would not form a closed loop. The issue of how to deal with intrinsic curvature is discussed in [27].
} 
Collecting terms, we can now express the gravitational part of the constraint as:

$$
\begin{aligned}
C_{\text {grav }}= & -4\left(8 \pi \gamma^{3} \mu_{o}^{3} G\right)^{-1} \sum_{i j k} \epsilon^{i j k} \operatorname{tr}\left(h_{i}^{\left(\mu_{o}\right)} h_{j}^{\left(\mu_{o}\right)} h_{i}^{\left(\mu_{o}\right)-1} h_{j}^{\left(\mu_{o}\right)-1} h_{k}^{\left(\mu_{o}\right)}\left\{h_{k}^{\left(\mu_{o}\right)-1}, V\right\}\right) \\
& +O\left(c^{3} \mu_{o}\right)
\end{aligned}
$$

where, the term proportional to identity in the leading contribution to $F_{a b}^{i}$ in (30) drops out because of the trace operation and where we used $\epsilon^{a b c}{ }^{o} \omega_{a}^{i o} \omega_{b}^{j o} \omega_{c}^{k}=\sqrt{{ }^{o} q} \epsilon^{i j k}$. Note that, in contrast to the situation with triads in section 3.3, now the dependence on $\mu_{o}$ does not drop out. However, one can take the limit $\mu_{o} \rightarrow 0$. Using the explicit form of the holonomies $h_{i}^{\left(\mu_{o}\right)}$, one can verify that the leading term in (34) has a well-defined limit which equals precisely the classical constraint. Thus, now $\mu_{o}-$ or the length of the edge used while expressing $F_{a b}$ in terms of the holonomy around the square $\alpha_{i j}$ - plays the role of a regulator. Because of the presence of the curvature term, there is no natural way to express the constraint exactly in terms of our elementary variables; a limiting procedure is essential. This faithfully mirrors the situation in the full theory: there, again, the curvature term is recovered by introducing small loops at vertices of graphs and the classical expression of the constraint is recovered only in the limit in which the loop shrinks to zero.

Let us focus on the leading term in (34). As in the full theory, this term is manifestly finite and can be promoted to a quantum operator directly. The resulting regulated constraint is:

$$
\begin{aligned}
\hat{C}_{\text {grav }}^{\left(\mu_{o}\right)}= & 4 i\left(\gamma^{3} \mu_{o}^{3} \ell_{\mathrm{Pl}}^{2}\right)^{-1} \sum_{i j k} \epsilon^{i j k} \operatorname{tr}\left(\hat{h}_{i}^{\left(\mu_{o}\right)} \hat{h}_{j}^{\left(\mu_{o}\right)} \hat{h}_{i}^{\left(\mu_{o}\right)-1} \hat{h}_{j}^{\left(\mu_{o}\right)-1} \hat{h}_{k}^{\left(\mu_{o}\right)}\left[\hat{h}_{k}^{\left(\mu_{o}\right)-1}, \hat{V}\right]\right) \\
= & 96 i\left(\gamma^{3} \mu_{o}^{3} \ell_{\mathrm{Pl}}^{2}\right)^{-1} \sin ^{2} \frac{\mu_{o} c}{2} \cos ^{2} \frac{\mu_{o} c}{2} \\
& \times\left(\sin \frac{\mu_{o} c}{2} \hat{V} \cos \frac{\mu_{o} c}{2}-\cos \frac{\mu_{o} c}{2} \hat{V} \sin \frac{\mu_{o} c}{2}\right)
\end{aligned}
$$

Its action on the eigenstates of $\hat{p}$ is

$$
\hat{C}_{\text {grav }}^{\left(\mu_{o}\right)}|\mu\rangle=3\left(\gamma^{3} \mu_{o}^{3} \ell_{\mathrm{Pl}}^{2}\right)^{-1}\left(V_{\mu+\mu_{o}}-V_{\mu-\mu_{o}}\right)\left(\left|\mu+4 \mu_{o}\right\rangle-2|\mu\rangle+\left|\mu-4 \mu_{o}\right\rangle\right) .
$$

On physical states, this action must equal that of the matter Hamiltonian $-8 \pi G \hat{C}_{\text {matter. }}$

Now, the limit $\mu_{o} \rightarrow 0$ of the classical expression (34) exists and equals the classical Hamiltonian constraint which, however, contains $c^{2}$ (see (11)). 
Consequently, the naive limit of the operator $\hat{C}_{\text {grav }}^{\left(\mu_{o}\right)}$ also contains $\hat{c}^{2}$. However, since $\hat{c}^{2}$ is not well-defined on $\mathcal{H}_{\text {grav }}^{S}$, now the limit as $\mu_{o} \rightarrow 0$ fails to exist. Thus, we can not remove the regulator in the quantum theory of the reduced model. In the full theory, by contrast, one can remove the regulator and obtain a well-defined action on diffeomorphism invariant states [14]. This difference can be directly traced back to the assumption of homogeneity. ${ }^{9}$ In the full theory, there is nonetheless a quantization ambiguity associated with the choice of the $j$ label used on the new edges introduced to define the operator corresponding to $F_{a b}$ [29]. That is, in the full theory, the quantization procedure involves a pair of labels $(e, j)$ where $e$ is a continuous label denoting the new edge and $j$ is a discrete label denoting the spin on that edge. Diffeomorphism invariance ensures that the quantum constraint is insensitive to the choice of $e$ but the dependence on $j$ remains as a quantization ambiguity. In the reduced model, diffeomorphism invariance is lost and the pair $(e, j)$ of the full theory collapses into a single continuous label $\mu_{o}$ denoting the length of the edge introduced to define $F_{a b}$. The dependence on $\mu_{o}$ persists - there is again a quantization ambiguity but it is now labelled by a continuous label $\mu_{o}$. Thus, comparison of the situation with that in the full theory suggests that we should not regard $\hat{C}_{\text {grav }}^{\left(\mu_{o}\right)}$ as an approximate quantum constraint; it is more appropriate to think of the $\mu_{o}$-dependence in (35) as a quantization ambiguity in the exact quantum constraint. This is the viewpoint adopted in loop quantum cosmology.

If one works in the strict confines of the reduced model, there does not appear to exist a natural way of removing this ambiguity. In the full theory, on the other hand, one can fix the ambiguity by assigning the lowest nontrivial $j$ value, $j=1 / 2$, to each extra loop introduced to determine the operator analog of $F_{a b}$. This procedure can be motivated by the following heuristics. In the classical theory, we could use a loop enclosing an arbitrarily small area in the $a-b$ plane to determine $F_{a b}$ locally. In quantum geometry, on the other hand, the area operator (of an open surface) has a lowest eigenvalue $a_{o}=(\sqrt{3} \gamma) / 4 \ell_{\mathrm{Pl}}^{2}[17,30]$ suggesting that it is physically inappropriate to try to localize $F_{a b}$ on arbitrarily small surfaces. The best one could do is to

\footnotetext{
${ }^{9}$ In the full theory, one triangulates the manifold with tetrahedra of coordinate volume $\mu_{o}^{3}$ and writes the integral $C(N):=\int d^{3} x N \epsilon_{i j k} F_{a b}^{i} e^{-1} E^{a j} E^{b k}$ as the limit of a Riemann sum, $C(N)=\lim _{\mu_{o} \rightarrow 0} \sum \mu_{o}^{3} N \epsilon_{i j k} F_{a b}^{i} e^{-1} E^{a j} E^{b k}$, where the sum is over tetrahedra. If we now replace $F$ by a holonomy around a square $\alpha$ of length $\mu_{o}, F \sim \mu_{o}^{-2}\left(h_{\alpha}-1\right)$, and the triad term by a Poisson bracket, $e^{-1} E E \sim \mu_{o}^{-1} h\left\{h^{-1}, V\right\}$, and pass to quantum operators, we obtain $\hat{C}(N) \sim \lim \sum \operatorname{tr} \hat{h}_{\alpha} \hat{h}\left[\hat{h}^{-1}, \hat{V}\right]$. The $\mu_{o}$ factors cancel out but, in the sum, the number of terms goes to infinity as $\mu_{o} \rightarrow 0$. However, the action of the operator on a state based on any graph is non-trivial only at the vertices of the graph whence only a finite number of terms in the sum survive and these have a well defined limit on diffeomorphism invariant states. In the reduced model, because of homogeneity, all terms in the sum contribute equally and hence the sum diverges.
} 
consider a loop spanning an area $a_{o}$, consider the holonomy around the loop to determine the integral of $F_{a b}$ on a surface of area $a_{o}$, and then extract an effective, local $F_{a b}$ by setting the integral equal to $a_{o} F_{a b}$. It appears natural to use the same physical considerations to remove the quantization ambiguity also in the reduced model. Then, we are led to set the area of the smallest square spanned by $\alpha_{i j}$ to $a_{o}$, i.e. to set $\left(\gamma \mu_{o}\right) \ell_{\mathrm{Pl}}^{2}=a_{o}$, or $\mu_{o}=\sqrt{3} / 4$. Thus, while in the reduced model itself, area eigenvalues can assume arbitrarily small values, if we 'import' from the full theory the value of the smallest non-zero area eigenvalue, we are naturally led to set $\mu_{o}=\sqrt{3} / 4$. We will do so.

To summarize, in loop quantum cosmology, we adopt the viewpoint that (35), with $\mu_{o}=\sqrt{3} / 4$, is the 'fundamental' Hamiltonian constraint operator which 'correctly' incorporates the underlying discreteness of quantum geometry and the classical expression (11) is an approximation which is valid only in regimes where this discreteness can be ignored and the continuum picture is valid. We will justify this assertion in section 4.3 .

\subsection{Physical states}

Let us now solve the quantum constraint and obtain physical states. For simplicity, we assume that the matter is only minimally coupled to gravity (i.e., there are no curvature couplings). As in general non-trivially constrained systems, one expects that the physical states would fail to be normalizable in the kinematical Hilbert space $\mathcal{H}^{S}=\mathcal{H}_{\text {grav }}^{S} \otimes \mathcal{H}_{\text {matter }}^{S}$ (see, e.g., $[31,18]$ ). However, as in the full theory, they do have a natural 'home'. We again have a triplet

$$
\mathrm{Cyl}_{S} \subset \mathcal{H}^{S} \subset \mathrm{Cyl}_{S}^{\star}
$$

of spaces and physical states will belong to $\mathrm{Cyl}_{S}^{\star}$, the algebraic dual of $\mathrm{Cyl}_{S}$. Since elements of $\mathrm{Cyl}_{S}^{\star}$ need not be normalizable, we will denote them by $(\Psi \mid$. (The usual, normalizable bras will be denoted by $\langle\Psi|$.)

It is convenient to exploit the existence of a triad representation. Then, every element $\left(\Psi \mid\right.$ of $\mathrm{Cyl}_{S}^{\star}$ can be expanded as

$$
\left(\Psi \mid=\sum_{\mu} \psi(\phi, \mu)\langle\mu|\right.
$$

where $\phi$ denotes the matter field and $\langle\mu|$ are the (normalized) eigenbras of $\hat{p}$. Note that the sum is over a continuous variable $\mu$ whence $(\Psi \mid$ need not be normalizable. Now, the constraint equation

$$
\left(\Psi \mid\left(\hat{C}_{\text {grav }}^{\left(\mu_{o}\right)}+8 \pi G \hat{C}_{\text {matter }}^{\left(\mu_{o}\right)}\right)^{\dagger}=0\right.
$$


turns into the equation

$$
\begin{gathered}
\left(V_{\mu+5 \mu_{o}}-V_{\mu+3 \mu_{o}}\right) \psi\left(\phi, \mu+4 \mu_{o}\right)-2\left(V_{\mu+\mu_{o}}-V_{\mu-\mu_{o}}\right) \psi(\phi, \mu) \\
+\quad\left(V_{\mu-3 \mu_{o}}-V_{\mu-5 \mu_{o}}\right) \psi\left(\phi, \mu-4 \mu_{o}\right)=-\frac{1}{3} 8 \pi G \gamma^{3} \mu_{o}^{3} \ell_{\mathrm{Pl}}^{2} \hat{C}_{\text {matter }}^{\left(\mu_{o}\right)}(\mu) \psi(\phi, \mu)
\end{gathered}
$$

for the coefficients $\psi(\phi, \mu)$, where $\hat{C}_{\text {matter }}^{\left(\mu_{o}\right)}(\mu)$ only acts on the matter fields (and depends on $\mu$ via metric components in the matter Hamiltonian). Note that, even though $\mu$ is a continuous variable, the quantum constraint is a difference equation rather than a differential equation. Strictly, (39) just constrains the coefficients $\psi(\phi, \mu)$ to ensure that $(\Psi \mid$ is a physical state. However, since each $\langle\mu|$ is an eigenbra of the volume operator, it tells us how the matter wave function is correlated with volume, i.e., geometry. Now, if one wishes, one can regard $p$ as providing a heuristic 'notion of time', and then think of (39) as an evolution equation for the quantum state of matter with respect to this time. (Note that $p$ goes from $-\infty$ to $\infty$, negative values corresponding to triads which are oppositely oriented to the fiducial one. The classical big-bang corresponds to $p=0$.) While this heuristic interpretation often provides physical intuition for (39) and its consequences, it is not essential for what follows; one can forego this interpretation entirely and regard (39) only as a constraint equation.

What is the fate of the classical singularity? At the big bang, the scale factor goes to zero. Hence it corresponds to the state $|\mu=0\rangle$ in $\mathcal{H}_{\text {grav }}^{S}$. So, the key question is whether the quantum 'evolution' breaks down at $\mu=0$. Now, the discrete 'evolution equation' (39) is essentially the same as that considered in the first papers on isotropic loop quantum cosmology $[2,3]$ and that discussion implies that the quantum physics does not stop at the big-bang.

For completeness, we now recall the main argument. The basic idea is to explore the key consequences of the difference equation (39) which determine what happens at the initial singularity. Starting at $\mu=-4 N \mu_{o}$ for some large positive $N$, and fixing $\psi\left(\phi,-4 N \mu_{o}\right)$ and $\psi\left(\phi,(-4 N+4) \mu_{o}\right)$, one can use the equation to determine the coefficients $\psi\left(\phi,(-4 N+4 n) \mu_{0}\right)$ for all $n>1$, provided the coefficient of the highest order term in (39) continues to remain non-zero. Now, it is easy to verify that the coefficient vanishes if and only if $n=N$. Thus, the coefficient $\psi(\phi, \mu=0)$ remains undetermined. In its place, we just obtain a consistency condition constraining the coefficients $\psi(\phi, \mu=$ $-4)$ and $\psi(\phi, \mu=-8)$. Now, since $\psi(\phi, \mu=0)$ remains undetermined, at first sight, it may appear that we can not 'evolve' past the singularity, i.e. that the quantum evolution also breaks down at the big-bang. However, the main point is that this is not the case. For, the coefficient $\psi(\phi, \mu=$ 0 ) just decouples from the rest. This comes about because, as a detailed 
examination shows, the minimally coupled matter Hamiltonians annihilate $\psi(\phi, \mu)$ for $\mu=0[1,25]$ and $V_{\mu_{o}}=V_{-\mu_{o}}$. Thus, unlike in the classical theory, evolution does not stop at the singularity; the difference equation (39) lets us 'evolve' right through it. In this analysis, we started at $\mu=-4 N \mu_{o}$ because we wanted to test what happens if one encounters the singularity 'head on'. If one begins at a generic $\mu$, the 'discrete evolution' determined by (39) just 'jumps' over the classical singularity without encountering any subtleties.

To summarize, two factors were key to the resolution of the big bang singularity: i) as a direct consequence of quantum geometry, the Hamiltonian constraint is now a difference equation rather than a differential equation as in geometrodynamics; and ii) the coefficients in the difference equation are such that one can evolve unambiguously 'through' the singularity even though the coefficient $\psi(\phi, \mu=0)$ is undetermined. Both these features are robust: they are insensitive to factor ordering ambiguities and persist in more complicated cosmological models $[6,7]$.

Next, let us consider the space of solutions. An examination of the classical degrees of freedom suggests that the freedom in physical quantum states should correspond to two functions just of matter fields $\phi$. The space of solutions to the Hamiltonian constraint, on the other hand is much larger: there are as many solutions as there are functions $\psi(\phi, \mu)$ on an interval $\left[\mu^{\prime}-4 \mu_{o}, \mu^{\prime}+4 \mu_{o}\right)$, where $\mu^{\prime}$ is any fixed number. This suggests that a large number of these solutions may be redundant. Indeed, to complete the quantization procedure, one needs to introduce an appropriate inner product on the space of solutions to the Hamiltonian constraint. The physical Hilbert space is then spanned by just those solutions to the quantum constraint which have finite norm. In simple examples one generally finds that, while the space of solutions to all constraints can be very large, the requirement of finiteness of norm suffices to produce a Hilbert space of the physically expected size.

For the reduced system considered here, we have a quantum mechanical system with a single constraint in quantum cosmology. Hence it should be possible to extract physical states using the group averaging technique of the 'refined algebraic quantization framework' [31, 18, 32]. However, this analysis is yet to be carried out explicitly and therefore we do not yet have a good control on how large the physical Hilbert space really is. This issue is being investigated.

The Hamiltonian constraint equation differs markedly from the WheelerDeWitt equation of geometrodynamics in the Planck regime because it crucially exploits the discreteness underlying quantum geometry. But one might 
expect that in the continuum limit $\mu_{o} \rightarrow 0-$ which, from the quantum geometry perspective, is physically fictitious but nonetheless mathematically interesting - the present quantum constraint equation would reduce to the Wheeler-DeWitt equation. We will conclude this sub-section by showing that this expectation is indeed correct in a precise sense.

To facilitate this comparison, it is convenient to introduce some notation. Let us set

$$
p=\frac{1}{6} \gamma \mu \ell_{\mathrm{Pl}}^{2} .
$$

Then, the Wheeler-DeWitt equation can be written as

$$
\hat{C}_{\mathrm{grav}}^{\mathrm{wdw}} \psi(\phi, p):=\frac{2}{3} \ell_{\mathrm{Pl}}^{4}[\sqrt{|p|} \psi(\phi, p)]^{\prime \prime}=8 \pi G \hat{C}_{\mathrm{matter}}(p) \psi(\phi, p),
$$

where the prime denotes derivative with respect to $p$. If we now further set

$$
\tilde{\psi}(p):=\frac{1}{6} p_{o}^{-1}\left(V_{6\left(p+p_{o}\right) / \gamma \ell_{\mathrm{P} 1}^{2}}-V_{6\left(p-p_{o}\right) / \gamma \ell_{\mathrm{Pl}}^{2}}\right) \psi(p),
$$

with $p_{o}=\gamma \ell_{\mathrm{Pl}}^{2} \mu_{o} / 6$, our quantum constraint (39) reduces to:

$$
\begin{aligned}
\hat{\tilde{C}}_{\text {grav }}^{\left(\mu_{o}\right)} \tilde{\psi}(\phi, p) & :=-\frac{1}{12} \ell_{\mathrm{Pl}}^{4} p_{o}^{-2}\left(\tilde{\psi}\left(\phi, p+4 p_{o}\right)-2 \tilde{\psi}(\phi, p)+\tilde{\psi}\left(\phi, p-4 p_{o}\right)\right) \\
& =8 \pi G \hat{C}_{\text {matter }}(p) \psi(\phi, p)
\end{aligned}
$$

From now on we will consider only those 'wave functions' $\tilde{\psi}(\phi, p)$ which are smooth (more precisely, $C^{4}$ ) in their $p$ dependence. Then, it follows that

$$
\begin{aligned}
\hat{C}_{\text {grav }}^{\mathrm{wdw}} \psi(\phi, p) & =\hat{\tilde{C}}_{\text {grav }}^{\left(\mu_{o}\right)} \tilde{\psi}(\phi, p)+\ell_{\mathrm{Pl}}^{4} O\left(p_{o}^{2}\right) \tilde{\psi}^{\prime \prime \prime \prime}(\phi, p) \\
& +\ell_{\mathrm{Pl}}^{4} O\left(\frac{p_{o}^{2}}{p^{2}}\right) \tilde{\psi}^{\prime \prime}(\phi, p)+\ell_{\mathrm{Pl}}^{4} O\left(\frac{p_{o}^{2}}{p^{3}}\right) \tilde{\psi}^{\prime}(\phi, p)+\ell_{\mathrm{Pl}}^{4} O\left(\frac{p_{o}^{2}}{p^{4}}\right) \tilde{\psi}(\phi, p)
\end{aligned}
$$

Hence, in the limit $p_{o} \rightarrow 0$ (i.e., $\mu_{o} \rightarrow 0$ ), we have

$$
\hat{\tilde{C}}_{\mathrm{grav}}^{\left(\mu_{o}\right)} \tilde{\psi}(\phi, p) \mapsto \hat{C}_{\mathrm{grav}}^{\mathrm{wdw}} \psi(\phi, p)
$$

whence the discrete equation (41) reduces precisely to the Wheeler-DeWitt equation (40). Put differently, it has turned out that (41) is a well-defined discretization of (40).

One can also ask a related but distinct question: Is there a sense in which solutions to the Wheeler-DeWitt equation are approximate solutions to the 'fundamental' discrete evolution equation? The answer is again in the affirmative. Let us restrict ourselves to the part of the $p$-line where $p \gg p_{o}$, 
i.e., where the quantum volume of the universe is very large compared to the Planck scale. Consider the restriction, to this region, of a smooth solution $\psi(\phi, p)$ to $(40)$ and assume that it is slowly varying at the Planck scale in the sense that $\tilde{\psi} / \tilde{\psi}^{\prime} \sim s, \tilde{\psi} / \tilde{\psi}^{\prime \prime} \sim s^{2}$, etc, with $p_{o} \ll s \leq p$. Then, $\tilde{\psi}(\phi, p)$ is an approximate solution to the 'fundamental' quantum constraint (41) in the sense that:

$$
\begin{aligned}
& {\left[1+O\left(\frac{p_{o}^{2}}{s^{2}}\right)+O\left(\frac{p_{o}^{2}}{p^{2}}\right)+O\left(\frac{p_{o}^{2} s}{p^{3}}\right)+O\left(\frac{p_{o}^{2} s^{2}}{p^{4}}\right)\right] \hat{\tilde{C}}_{\text {grav }}^{\left(\mu_{o}\right)} \tilde{\psi}(\phi, p)} \\
& =8 \pi G \hat{C}_{\text {matter }}(p) \psi(\phi, p) .
\end{aligned}
$$

Note that, in contrast to the discussion about the relation between the two equations, we can not take the limit $p_{o} \rightarrow 0$ because we are now interested in the discrete evolution. The solution to the Wheeler-DeWitt equation is an approximate solution to the fundamental equation only to the extent that terms of the order $O\left(p_{o}^{2} / s^{2}\right), O\left(p_{o}^{2} / p^{2}\right), O\left(p_{o}^{2} s / p^{3}\right), O\left(p_{o}^{2} s^{2} / p^{4}\right)$ are negligible.

We will conclude with three remarks.

1) We saw in section 4.1 that the $\mu_{o} \rightarrow 0$ limit of the quantum constraint operator $\hat{C}_{\text {grav }}^{\left(\mu_{o}\right)}$ does not exist on $\mathcal{H}_{\text {grav }}^{S}$. Yet, in the above discussion of the 'continuum limit', we were able to take this limit. The resolution of this apparent paradox is that the limit is taken on a certain sub-space of $\mathrm{Cyl}^{\star}$, consisting of smooth functions of $p$ and none of these states belong to $\mathcal{H}_{\text {grav }}^{S}$. Indeed, since elements of $\mathcal{H}_{\text {grav }}^{S}$ have to be normalizable with respect to the inner product (17), they can have support only on a countable number of points; they cannot even be continuous. In particular, solutions to the Wheeler-DeWitt equation can not lie in $\mathcal{H}^{S}$; they belong only to the enlargement $\mathrm{Cyl}^{\star}$ of $\mathcal{H}^{S}$.

2) There is a close mathematical similarity between quantum cosmology discussed here and the 'polymer particle' example discussed in [22]. In that example, following the loop quantum gravity program, a new representation of the Weyl algebra is introduced for a point particle in non-relativistic quantum mechanics. In this representation, the Weyl operators are unitarily implemented but weak continuity, assumed in the Von Neumann uniqueness theorem, is violated for one of the two 1-parameter unitary groups. As a result (although the position operator exists) the momentum operator - the generator of infinitesimal space translations - fails to exist. This is meant to reflect the underlying discreteness of geometry. The 'fundamental' quantum evolution is given by a difference equation. But there is a precise sense in which the standard Schrödinger evolution is recovered in the regime of validity of non-relativistic quantum mechanics. The 'fundamental' discrete 
evolution is analogous to the present 'fundamental' quantum constraint (41) while the Schrödinger equation is the analog of the Wheeler-DeWitt equation (40). Therefore, details of the polymer particle analysis provide good intuition for the 'mechanism' that allows loop quantum cosmology to be very different from the standard one in the Planck regime and yet agree with it when the universe is large compared to the Planck scale.

3) As mentioned in section 1, in this paper we do not address the difficult issue of systematically deriving quantum cosmology from full loop quantum gravity. Indeed, since $\mathrm{Cyl}_{S} \not \subset \mathrm{Cyl}$, at first it seems it would be difficult to relate the two theories. However, note that the physical states of the symmetry reduced model are elements of $\mathrm{Cyl}_{S}^{\star}$ while those of the full theory are elements of $\mathrm{Cyl}^{\star}$, and $\mathrm{Cyl}_{S}^{\star}$ is contained in $\mathrm{Cyl}^{\star}$ : elements of $\mathrm{Cyl}_{S}^{\star}$ are those distributions on the full quantum configuration space $\overline{\mathcal{A}}$ which are supported only on the subspace $\overline{\mathcal{A}}_{S}$ of symmetric connections [33]. In particular, solutions to the quantum constraint discussed in this section do belong to $\mathrm{Cyl}^{\star}$. Therefore, it should be possible to recover such states by first considering the full quantum theory and then carrying out a symmetry reduction.

\subsection{Classical limit}

In section 4.1, we found that the gravitational part of the Hamiltonian constraint could not be introduced by a straightforward 'quantization' of the classical constraint (11) because there is no direct operator analog of $c$ on $\mathcal{H}_{\text {grav }}^{S}$. We then followed the strategy adopted in the full theory to arrive at the expression (35) of $\hat{C}_{\text {grav }}^{\left(\mu_{o}\right)}$. To ensure that this is a viable quantization, we need to show that (35) does reduce to (11) in the classical limit. In this sub-section, we will carry out this task.

For this purpose we will use coherent states peaked at points $\left(c_{o}, N \mu_{o}\right)$ of the classical phase space where $N \gg 1$ (i.e. the volume of the universe is very large compared to the Planck volume) and $c_{o} \ll 1$ (late times, when the extrinsic curvature is small compared to the fiducial scale $V_{o}^{-1 / 3}$ ). At such large volumes and late times, one would expect quantum corrections to become negligible. The question then is whether the expectation value of the quantum constraint $\hat{C}_{\text {grav }}^{\left(\mu_{o}\right)}$ in these coherent states equals the classical constraint (11) modulo negligible corrections. If so, $\hat{C}_{\text {grav }}^{\left(\mu_{o}\right)}$ would have the correct classical limit.

To construct a coherent state, we also have to specify the width $d$ of the Gaussian (i.e., 'tolerance' for quantum fluctuations of $p$ ). Now, since the 
quantum fluctuations in the volume of the universe must be much smaller than the volume itself, $d \ll N \mu_{o}$ and since we also want the uncertainty in $c$ to be small, we must have $\mu_{o} \ll d$. A coherent state of the desired type is then given by:

$$
|\Psi\rangle=\sum_{n}\left[\exp \left(-\left((n-N)^{2} \frac{\mu_{o}^{2}}{2 d^{2}}\right) \exp \left(-i\left((n-N) \mu_{o}\right) \frac{c_{o}}{2}\right)\right]\left|n \mu_{o}\right\rangle\right.
$$

(More precisely, $|\Psi\rangle$ is the 'shadow' on the regular lattice $\mu=n \mu_{o}$ of the coherent state in $\mathrm{Cyl}^{\star}$ uniquely selected by the triplet $\left(c_{o}, N \mu_{o}, d\right)$. For details, see [22], Section 4.) Our task is to compute the expectation value

$$
\left\langle\hat{C}_{\text {grav }}^{\left(\mu_{o}\right)}\right\rangle=\frac{\left\langle\Psi\left|\hat{C}_{\text {grav }}^{\left(\mu_{o}\right)}\right| \Psi\right\rangle}{\langle\Psi \mid \Psi\rangle}
$$

of the constraint operator (41):

$$
\hat{C}_{\text {grav }}^{\left(\mu_{o}\right)}|\mu\rangle=3\left(\gamma^{3} \mu_{o}^{3} \ell_{\mathrm{Pl}}^{2}\right)^{-1}\left(V_{\mu+\mu_{o}}-V_{\mu-\mu_{o}}\right)\left(\left|\mu+4 \mu_{o}\right\rangle-2|\mu\rangle+\left|\mu-4 \mu_{o}\right\rangle\right) .
$$

Let us first calculate the expectation value. Setting $\epsilon:=\mu_{o} / d$, we have:

$$
\begin{aligned}
\langle\Psi|\hat{C}| \Psi\rangle= & \sum_{n, n^{\prime}} \exp \left(-\frac{1}{2} \epsilon^{2}\left(\left(n^{\prime}-N\right)^{2}+(n-N)^{2}\right)\right) e^{i \frac{c_{o}}{2}\left(n^{\prime}-n\right) \mu_{o}}\left\langle n^{\prime} \mu_{o}|\hat{C}| n \mu_{o}\right\rangle \\
= & 3\left(\gamma^{3} \mu_{o}^{3} \ell_{\mathrm{Pl}}^{2}\right)^{-1} \sum_{n, n^{\prime}} \exp \left(-\frac{1}{2} \epsilon^{2}\left(\left(n^{\prime}-N\right)^{2}+(n-N)^{2}\right)\right) e^{i \frac{c_{o}}{2}\left(n^{\prime}-n\right) \mu_{o}} \\
& \times\left(V_{(n+1) \mu_{o}}-V_{(n-1) \mu_{o}}\right)\left\langlen ^ { \prime } \mu _ { o } \left|\left(\left|(n+4) \mu_{o}\right\rangle-2\left|n \mu_{o}\right\rangle+\left|(n-4) \mu_{o}\right\rangle\right)\right.\right. \\
= & 3\left(\gamma^{3} \mu_{o}^{3} \ell_{\mathrm{Pl}}^{2}\right)^{-1}\left[e^{2 i c_{o} \mu_{o}} \sum_{n} \exp \left(-\frac{1}{2} \epsilon^{2}\left((n+4-N)^{2}+(n-N)^{2}\right)\right)\right. \\
& \quad-2 \sum_{n} \exp \left(-V_{(n+1) \mu_{o}} \epsilon^{2}(n-N)^{2}\right)\left(V_{(n+1) \mu_{o}}\right) \\
& +e^{-2 i c_{o} \mu_{o}} \sum_{n} \exp \left(-\frac{1}{2} \epsilon^{2}\left((n-4-N)^{2}+(n-N)^{2}\right)\right) \\
& \left.\times\left(V_{(n+1) \mu_{o}}-V_{(n-1) \mu_{o}}\right)\right]
\end{aligned}
$$

To simplify this expression further, we note that all three sums in this expression are of the same form and focus on the first:

$$
\begin{aligned}
\sum_{n} \exp \left(-\frac{1}{2} \epsilon^{2}\left((n+4-N)^{2}\right.\right. & \left.\left.+(n-N)^{2}\right)\right)\left(V_{(n+1) \mu_{o}}-V_{(n-1) \mu_{o}}\right) \\
& =e^{-4 \epsilon^{2}} \sum_{n} e^{-\epsilon^{2}(n-N)^{2}}\left(V_{(n-1) \mu_{o}}-V_{(n-3) \mu_{o}}\right)
\end{aligned}
$$


where we have completed the square in the exponential and shifted the summation index by 2. To compute this sum, as in [22], we use the Poisson resummation formula

$$
\sum_{n} e^{-\epsilon^{2}(n-N)^{2}} f(n)=\sum_{n} \int e^{-\epsilon^{2}(y-N)^{2}} f(y) e^{2 \pi i y n} d y .
$$

This integral can be evaluated using the steepest descent approximation (see Appendix). One obtains:

$$
\begin{aligned}
\sum_{n} e^{-\epsilon^{2}(n-N)^{2}} f(n) & =\frac{\sqrt{\pi}}{\epsilon} \sum_{n} f\left(N+\frac{i \pi n}{\epsilon^{2}}\right) e^{-\frac{\pi^{2} n^{2}}{\epsilon^{2}}+2 \pi i n N}\left(1+O\left((N \epsilon)^{-2}\right)\right) \\
& =\frac{\sqrt{\pi}}{\epsilon} f(N)\left(1+O\left(e^{-\pi^{2} / \epsilon^{2}}\right)+O\left((N \epsilon)^{-2}\right)\right)
\end{aligned}
$$

where, in the last step, we used the fact that, since $\epsilon \ll 1$, terms with $n \neq 0$ are suppressed by the exponential. (Note that $N \epsilon \gg 1$ because $N \mu_{o} \gg d$, i.e., the permissible quantum fluctuation in the volume of the universe is much smaller than the volume of the universe at the phase space point under consideration.)

Finally, we can collect terms to compute the expectation value (46). Using $\langle\Psi \mid \Psi\rangle=(\sqrt{\pi} / \epsilon)\left(1+O\left(e^{-\pi^{2} / \epsilon^{2}}\right)\right)$, we have

$$
\begin{aligned}
\langle\hat{C}\rangle_{S}= & 3\left(\gamma^{3} \mu_{o}^{3} \ell_{\mathrm{Pl}}^{2}\right)^{-1}\left[e^{-4 \epsilon^{2}} e^{2 i c_{o} \mu_{o}}\left(V_{(N-1) \mu_{o}}-V_{(N-3) \mu_{o}}\right)\right. \\
& \left.-2\left(V_{(N+1) \mu_{o}}-V_{(N-1) \mu_{o}}\right)+e^{-4 \epsilon^{2}} e^{-2 i c_{o} \mu_{o}}\left(V_{(N+3) \mu_{o}}-V_{(N+1) \mu_{o}}\right)\right] \\
& \times\left(1+O\left(e^{-\pi^{2} / \epsilon^{2}}\right)+O\left((N \epsilon)^{-2}\right)\right) \\
= & \frac{1}{2}\left(\gamma^{2} \mu_{o}^{2}\right)^{-1} \sqrt{\gamma \mu_{o} \ell_{\mathrm{Pl}}^{2} / 6}\left[e^{-4 \epsilon^{2}} e^{2 i c_{o} \mu_{o}}\left((N-1)^{\frac{3}{2}}-(N-3)^{\frac{3}{2}}\right)\right. \\
& \left.-2\left((N+1)^{\frac{3}{2}}-(N-1)^{\frac{3}{2}}\right)+e^{-4 \epsilon^{2}} e^{-2 i c_{o} \mu_{o}}\left((N+3)^{\frac{3}{2}}-(N+1)^{\frac{3}{2}}\right)\right] \\
& \times\left(1+O\left(e^{-\pi^{2} / \epsilon^{2}}\right)+O\left((N \epsilon)^{-2}\right)\right) \\
= & \frac{3}{2}\left(\gamma^{2} \mu_{o}^{2}\right)^{-1} \sqrt{\gamma \mu_{o} \ell_{\mathrm{Pl}}^{2} N / 6}\left(e^{-4 \epsilon^{2}} e^{2 i c_{o} \mu_{o}}-2+e^{-4 \epsilon^{2}} e^{-2 i c_{o} \mu_{o}}\right) \\
& \times\left(1+O\left(e^{-\pi^{2} / \epsilon^{2}}\right)+O\left((N \epsilon)^{-2}\right)+O\left(N^{-1}\right)\right) \\
= & -6 \gamma^{-2} c_{o}^{2} \sqrt{P}\left(1+O\left((N \epsilon)^{-2}\right)+O\left(N^{-1}\right)+O\left(\epsilon^{2}\right)+O\left(c_{o}^{2}\right)\right)
\end{aligned}
$$

where we have set $P:=\frac{1}{6} \gamma \mu_{o} \ell_{\mathrm{Pl}}^{2} N$ and used the fact that $c_{o} \ll 1$ and $\mu_{o} \sim 1$ (we also dropped corrections of order $e^{-\pi^{2} / \epsilon^{2}}$ since they are always dwarfed by those of order $\epsilon^{2}$ ). Thus, the expectation value equals the classical constraint (11) up to small corrections of order $c_{o}^{2}, \ell_{\mathrm{Pl}}^{4} /(P \epsilon)^{2}, \ell_{\mathrm{Pl}}^{2} / P$ and $\epsilon^{2}$. (Note that each of them can dominate the other corrections depending on the values of the different parameters.) Hence, (35) is a viable quantization of the classical expression (11). 


\section{Discussion}

Let us begin with a brief summary of the main results. In section 2, we carried out a systematic symmetry reduction of the phase space of full general relativity in the connection variables. In the spatially flat model considered here, our connection coefficient $c$ equals the only non-trivial (i.e. dynamical) component of the extrinsic curvature (modulo a factor of $\gamma$ ) and our conjugate momentum $p$ equals the only non-trivial metric component (modulo $\operatorname{sgn} p$ ). Hence, our symmetry reduced Hamiltonian description is the same as that of geometrodynamics. By contrast, we saw in section 3 that quantum theories are dramatically different already at the kinematic level. In loop quantum cosmology, the Hilbert space $\mathcal{H}_{\text {grav }}^{S}$ is spanned by almost period functions of $c$ while in geometrodynamics it would be spanned by square-integrable functions of $c$. The intersection between the two Hilbert spaces is only the zero element! In loop quantum cosmology, the fundamental operators are $\hat{p}$ and $\hat{\mathcal{N}}_{\mu}=\operatorname{exp(i\mu c/2)}$; unlike in geometrodynamics, there is no operator corresponding to $c$ itself. Although $\hat{p}$ is unbounded and its spectrum consists of the entire real line, all its eigenvectors are normalizable and the Hilbert space is the direct sum of the 1-dimensional sub-spaces spanned by the eigenspaces. In geometrodynamics, on the other hand, none of the eigenvectors of $\hat{p}$ is normalizable; the Hilbert space is a direct integral of its 'eigenspaces'. This marked difference is responsible for the fact that, while the triad operator (which encodes the inverse of the scale factor) is unbounded in geometrodynamics, it is bounded in loop quantum cosmology. Consequently, in the state corresponding to the classical singularity, the curvature is large, but it does not diverge in loop quantum cosmology.

In section 4 we discussed the Hamiltonian constraint, i.e., quantum dynamics. Because there is no direct operator analog of $c$, we had to introduce the constraint operator $\hat{C}_{\text {grav }}^{\left(\mu_{o}\right)}$ by an indirect construction. Here, we followed the strategy used in the full theory [14], expressing curvature $F_{a b}^{i}$ of the gravitational connection $A_{a}^{i}$ in terms of its holonomies around suitable loops. In the full theory, one can take the limit as the loop shrinks to zero and obtain a well-defined operator on diffeomorphism invariant states. The reduced model, by contrast, fails to be diffeomorphism invariant and the operator diverges on $\mathcal{H}_{\text {grav }}^{S}$ in the limit. Therefore, to obtain a well-defined operator, we used loops enclosing an area $a_{o}$, the smallest non-zero quantum of area in quantum geometry. The resulting operator can be regarded as a 'good' quantization of the classical constraint function because it has the correct classical limit. The resulting quantum constraint equation has novel and physically appealing properties. First, it is a difference -rather than differential - equation and thus provides a 'discrete time evolution'. 
Second, the coefficients in this difference equation are such that the 'evolution' does not break down at the singularity; quantum physics does not stop at the big-bang! This occurs without fine tuning matter or making it violate energy conditions. Furthermore, while in consistent discrete models the singularity is often 'avoided' because discrete 'time steps' are such that one simply leaps over the point where the singularity is expected to occur [36], here, one can and does confront the singularity head on only to find that it has been resolved by the quantum 'evolution'. Furthermore, these features are robust $[6,7]$. However, near the big-bang, the state is 'extremely quantum mechanical,' with large fluctuations. Thus, the classical space-time 'dissolves' near the big-bang. In this regime, we can analyze the structure only in quantum mechanical terms; we can no longer use our classical intuition which is deeply rooted in space-times and small fluctuations around them.

In the Planck regime, the predictions of loop quantum cosmology are thus markedly different from those of standard quantum cosmology based on geometrodynamics. The origin of this difference can be traced back to the fact that while loop quantum cosmology makes a crucial use of the fundamental discreteness of quantum geometry, standard cosmology is based on a continuum picture. One would therefore expect that the difference between the two would become negligible in regimes in which the continuum picture is a good approximation. We established two results to show that this expectation is indeed correct. First, there is a precise sense in which the difference equation of loop quantum cosmology reduces to the Wheeler-DeWitt differential equation in the continuum limit. Second, in the regime far removed from the Planck scale, solutions to the Wheeler-DeWitt equation solve the difference equation to an excellent accuracy. Thus, the quantum constraint of loop quantum cosmology modifies the Wheeler-DeWitt equation in a subtle manner: the modification is significant only in the Planck regime and yet manages to be 'just right' to provide a natural resolution of the big-bang singularity.

Next, let us re-examine the early papers on loop quantum cosmology in terms of the precise mathematical framework developed in this paper. In the present terminology, in the previous discussion one effectively restricted oneself just to periodic functions $\exp (i n c / 2)$, rather than almost periodic functions $\exp (i \mu c / 2)$ considered here. Thus the gravitational Hilbert space $\mathcal{H}_{\text {grav }}^{S, P}$ considered there is the rather small, periodic sub-space of the present $\mathcal{H}_{\text {grav }}^{S}$. While this restriction did have heuristic motivation, it amounted to forcing $c$ to be periodic. ${ }^{10}$ From the geometrodynamical perspective, the

\footnotetext{
${ }^{10}$ In the symmetry reduction, one began with the general geometric theory of invariant connections and found that components of a homogeneous connection transform as scalars
} 
extrinsic curvature was made periodic (with a very large period) and it was then not surprising that the eigenvalues of the scale factor (and hence also the volume) operator could only be discrete. However, a careful analysis shows that the restriction to periodic functions can not be justified: periodic functions fail to separate the symmetric connections. Thus, in the earlier treatments, the space of configuration variables was 'too small' already at the classical level and this led to an artificial reduction in the size of the quantum state space.

In the analysis presented here, $c$ is not periodic. As a consequence, the spectrum of the volume operator is the entire real line. Yet, there is discreteness in a more subtle sense: all eigenvectors of the volume operator are normalizable. This is a direct consequence of the fundamental premise of loop quantum gravity that the quantum Hilbert space carries well defined operators corresponding to holonomies and not connections themselves. In the full theory, this feature does make the spectra of geometric operators discrete and their eigenvectors normalizable. Because of the homogeneity assumption, however, the first of these features is lost in loop quantum cosmology but the second does survive. The surprising and highly non-trivial fact is that this is sufficient for several of the main results of earlier papers to continue to hold: i) the inverse scale factor is still bounded from above; ii) the Hamiltonian constraint is again a difference equation; and, iii) the coefficients in this equation are such that the singularity is resolved in the quantum theory. Furthermore, the current analysis provided a systematic approach to verify that the constraint operator $\hat{C}_{\text {grav }}^{\left(\mu_{o}\right)}$ has the correct classical limit and made its relation to the Wheeler-DeWitt operator more precise and transparent. However, as in earlier papers, the issue of finding the inner product on physical states is yet to be analyzed in detail. While the group averaging procedure $[31,18,32]$ provides a natural avenue, a detailed implementation of this program has only begun. The issue of whether the 'pre-classicality' condition selects unique quantum states, thereby providing a natural solution to the issue of initial conditions can be addressed systematically only after one has a better control on the physical Hilbert space. Finally, the discussion of section 4.3 not only shows that the classical Einstein's equation is recovered in loop quantum cosmology in an appropriate limit but it also provides a systematic approach to the problem of finding quantum corrections to Einstein's equations. ${ }^{11}$ These corrections are now

under gauge transformations [34]. The appropriate, polymer theory for real-valued scalar fields was developed only recently [35] and requires, as in the present paper, the Bohr compactification of the real line.

${ }^{11}$ There appears to be a rather general impression that Einstein's equations are not modified in loop quantum gravity. As our discussion of 4.3 shows, this is not the case. It is true that we simply promoted the classical Hamiltonian constraint function to an operator. 
being worked out systematically.

We conclude with a general observation. The way in which the bigbang singularity is resolved has potentially deep implications on questions about the origin of the universe. For instance, the question of whether the universe had a beginning at a finite time is now 'transcended'. At first, the answer seems to be 'no' in the sense that the quantum evolution does not stop at the big bang. However, since space-time geometry 'dissolves' near the big-bang, there is no longer a notion of time, or of 'before' or 'after' in the familiar sense. Therefore, strictly, the question is no longer meaningful. The paradigm has changed and meaningful questions must now be phrased differently, without using notions tied to classical space-times. A similar shift of paradigm occurred already with the advent of general relativity. Before Einstein, philosophers argued that the universe could not have a finite beginning because, if it did, one could ask what there was before. However, this question pre-supposes that space-time is an eternal, passive arena and matter simply evolves in it. With general relativity, we learned that space and time are 'born with matter', whence the question of 'what was there before' is no longer meaningful. Loop quantum cosmology brings about a further shift of paradigm, weeding out certain questions that seemed meaningful in classical general relativity and requiring that they be replaced by more refined questions, formulated in the context of quantum space-times.

Acknowledgments: We thank the participants in the 'Quantum field theory on curved space-times' workshop at the Erwin Schrödinger Institute for stimulating discussions. We are especially grateful to Bill Unruh for raising several conceptual issues addressed here, and to Klaus Fredenhagen for the key observation that the Bohr compactification of the real line is the appropriate quantum configuration space. This work was supported in part by the NSF grant PHY-0090091, KBN grant 2P03B 2724, the Eberly research funds of Penn State and the Erwin Schrödinger Institute.

However, because there is no direct operator analog of $c$, this 'quantization' is subtle and even on semi-classical (coherent) states, sharply peaked at classical configurations, the expectation value of the constraint operator equals the classical constraint function with small but very specific quantum corrections. We expect that the same procedure can be applied in the full theory to obtain quantum corrections to full Einstein's equations. 


\section{A Method of Steepest Descent}

In this appendix, we will show that the method of steepest descent can be used to evaluate the right side of the Poisson resummation formula (47):

$$
\sum_{n} e^{-\epsilon^{2}(n-N)^{2}} f(n)=\sum_{n} \int e^{-\epsilon^{2}(y-N)^{2}} f(y) e^{2 \pi i y n} d y
$$

To begin with, we will assume that $f$ is analytic and return to the cases of interest at the end.

Note first that the Fourier integral can be written as

$$
\int e^{-\epsilon^{2}(y-N)^{2}} f(y) e^{2 \pi i y n} d y=N \int g(z) e^{N h_{n}(z)} d z,
$$

where $g(z)=f(N z)$ is the combination of volume eigenvalues, and

$$
h_{n}(z):=-\epsilon^{2} N(z-1)^{2}+2 \pi i n z .
$$

For large $N$, the integral can be evaluated by the method of steepest descent. For this, we have to first find the saddle points. In our case, this amounts to finding solutions $z_{0}$ of $h^{\prime}\left(z_{0}\right)=-2 \epsilon^{2} N\left(z_{0}-1\right)+2 \pi i n=0$. This equation has a single solution, $z_{0}=1+i \pi n / N \epsilon^{2}$. Steepest paths through $z_{0}$ are defined by constant imaginary part $\operatorname{Im} h_{n}(z)=\operatorname{Im} h_{n}\left(z_{0}\right)$. If we write $z=\xi+i \eta$ with real $\xi, \eta$, we obtain $-2 \epsilon^{2} N(\xi-1) \eta+2 \pi n \xi=2 \pi n$, which has the solutions $\xi=1$ or $\eta=\pi n / \epsilon^{2} N$. We can now deform the original integration along the real line to an integration along the steepest path $\eta=\pi n / \epsilon^{2} N$, i.e. $z=t+i \pi n / \epsilon^{2} N$ with $t$ real. Next, let us change the integration variable to $u$ by $t=1+u / \epsilon \sqrt{N}$ such that

$$
u^{2}=h_{n}\left(z_{0}\right)-h_{n}(z)=\epsilon^{2} N(t-1)^{2} .
$$

With $d z=d u / \epsilon \sqrt{N}$ we obtain

$$
\int g(z) e^{N h_{n}(z)} d z=\epsilon^{-1} N^{-\frac{1}{2}} \int_{-\infty}^{\infty} g\left(t(u)+i \pi n / \epsilon^{2} N\right) e^{N\left(h_{n}\left(z_{0}\right)-u^{2}\right)} d u
$$

So far, everything was exact. In order to be able to compute the integral, we now use the Taylor expansion:

$$
\begin{aligned}
g\left(t(u)+i \pi n / \epsilon^{2} N\right)= & g\left(1+u / \epsilon \sqrt{N}+i \pi n / \epsilon^{2} N\right)=g\left(1+i \pi n / \epsilon^{2} N\right) \\
& +u g^{\prime}\left(1+i \pi n / \epsilon^{2} N\right) / \epsilon \sqrt{N}+u^{2} g^{\prime \prime}\left(1+i \pi n / \epsilon^{2} N\right) / \epsilon^{2} N \\
& +O\left(u^{3} / \epsilon^{3} N^{\frac{3}{2}}\right)
\end{aligned}
$$


Then, the integral can be evaluated approximately as [37]:

$$
\begin{aligned}
\int g(z) e^{N h_{n}(z)} d z= & \epsilon^{-1} N^{-\frac{1}{2}}\left[g\left(1+i \pi n / \epsilon^{2} N\right) \int_{-\infty}^{\infty} e^{N\left(h_{n}\left(z_{0}\right)-u^{2}\right)} d u\right. \\
& +\epsilon^{-2} N^{-1} g^{\prime \prime}\left(1+i \pi n / \epsilon^{2} N\right) \int_{-\infty}^{\infty} u^{2} e^{N\left(h_{n}\left(z_{0}\right)-u^{2}\right)} d u \\
& \left.+O\left(\epsilon^{-4} N^{-2} \int_{-\infty}^{\infty} u^{4} e^{N\left(h_{n}\left(z_{0}\right)-u^{2}\right)} d u\right)\right] \\
= & \sqrt{\pi} \epsilon^{-1} N^{-1} g\left(1+i \pi n / \epsilon^{2} N\right) e^{-\pi^{2} n^{2} / \epsilon^{2}+2 \pi i n N} \\
& \times\left(1+O\left((N \epsilon)^{-2}\right)\right)
\end{aligned}
$$

where the corrections of order $(N \epsilon)^{-2}$ are small because $d \ll N \mu_{o}$. (We assumed $g^{\prime \prime}\left(z_{0}\right)$ to be of the same order as $g\left(z_{0}\right)$, which is the case for the functions we are interested in here.)

We can now return to the Poisson re-summation formula and compute the sum:

$$
\begin{aligned}
\sum_{n} e^{-\epsilon^{2}(n-N)^{2}} f(n)= & \sum_{n} \int e^{-\epsilon^{2}(y-N)^{2}} f(y) e^{2 \pi i y n} d y \\
= & N \sum_{n} \int f(N z) e^{N h_{n}(z)} d z \\
= & \sqrt{\pi} \epsilon^{-1} \sum_{n} f\left(N+i \pi n / \epsilon^{2}\right) e^{-\pi^{2} n^{2} / \epsilon^{2}+2 \pi i n N} \\
& \times\left(1+O\left((N \epsilon)^{-2}\right)\right) \\
= & \sqrt{\pi} \epsilon^{-1} f(N)\left(1+O\left(e^{-\pi^{2} / \epsilon^{2}}\right)+O\left((N \epsilon)^{-2}\right)\right)
\end{aligned}
$$

where, in the last step, we used the fact that terms with $n \neq 0$ are suppressed by the exponential because $\epsilon \ll 1$.

Finally, let us address the fact that the functions $f(y)$ of interest in the main text come from the eigenvalues of the volume operator which are of the type $V_{n \mu_{o}}=\left(\gamma|n| \mu_{o} / 6\right)^{3 / 2}$ and, because of the absolute value involved, $f(y)$ fail to be analytic in $y$ at $y=0$. One can circumvent this potential problem by replacing them with functions $\tilde{f}(y)$ which are the analytic continuations (to the upper half complex $y$-plane) of the restriction of $f(y)$ to the positive real $y$-axis and then carry out the above calculation for $\tilde{f}$ which, by construction, are analytic. (Thus, for example, $|y|^{3 / 2}$ is replaced by $\left(y^{2}\right)^{3 / 4}$.) The error, $\sum_{n} \exp \left(-\epsilon^{2}(n-N)^{2}\right)[f(n)-\tilde{f}(n)]$, can be shown to be of the order $O\left(e^{-(N \epsilon)^{2}}\right)$ which is negligible compared to the corrections derived above. The error is so small because the function integrated on the right side of the Poisson resummation formula is strongly peaked at a very large positive 
value $y=N$ of $y$ and the contribution from the negative half of the real $y$ axis is extremely small.

\section{References}

[1] M. Bojowald, Inverse scale factor in isotropic quantum geometry, Phys. Rev., D64 (2001), 084018.

[2] M. Bojowald, Loop quantum cosmology IV: Discrete time evolution Class. Quant. Grav., 18 (2001), 1071-1088.

[3] M. Bojowald, Absence of singularity in loop quantum cosmology, Phys. Rev. Lett., 86 (2001), 5227-5230.

[4] M. Bojowald, Dynamical initial conditions in quantum cosmology, Phys. Rev. Lett., 87 (2001), 121301.

[5] M. Bojowald, Inflation from quantum geometry, Phys. Rev. Lett., 89 (2002), 261301.

[6] M. Bojowald, Quantization ambiguities in isotropic quantum geometry, Class. Quantum Grav., 19 (2002), 5113-5130.

[7] M. Bojowald, Homogeneous loop quantum cosmology, Class. Quantum Grav., 20 (2003), 2595-2615.

[8] A. Ashtekar and C. J. Isham, Representation of the holonomy algebras of gravity and non-Abelian gauge theories, Class. Quant. Grav., 9 (1992), $1433-1467$.

[9] A. Ashtekar and J. Lewandowski, Representation theory of analytic holonomy algebras, in 'Knots and Quantum Gravity', edired by J.C. Baez, Oxford U. Press, Oxford 1994.

[10] J.C. Baez, J.C. Generalized measures in gauge theory, Lett. Math. Phys., 31 (1994), 213-223.

[11] D. Marolf and J. Mourão, On the support of the Ashtekar-Lewandowski measure, Commun. Math. Phys., 170 (1995), 583-606.

[12] A. Ashtekar and J. Lewandowski, Projective techniques and functional integration, Jour. Math. Phys., 36 (1995), 2170-2191.

[13] A. Ashtekar and J. Lewandowski, Differential geometry on the space of connections using projective techniques, Jour. Geo. \& Phys., 17 (1995), $191-230$. 
[14] Thiemann, T. (1996) Anomaly-free formulation of non-perturbative, four-dimensional Lorentzian quantum gravity, Phys. Lett., $\mathbf{B 3 8 0}$ (1996), 257-264;

Quantum spin dynamics (QSD), Class. Quant. Grav., 15 (1998), 839873 ;

QSD V: Quantum gravity as the natural regulator of matter quantum field theories, Class. Quant. Grav., 15 (1998), 1281-1314.

[15] A. Ashtekar and J. Lewandowski,Background independent quantum gravity: A status report (pre-print, 2003)

[16] A. Ashtekar, New variables for classical and quantum gravity, Phys. Rev. Lett., 57 (1986), 2244-2247;

New Hamiltonian formulation of general relativity, Phys. Rev., D36 (1987), 1587-1602.

[17] A. Ashtekar and J. Lewandowski, Quantum theory of geometry I: Area operators, Class. Quant. Grav., 14 (1997), A55-A81.

[18] A. Ashtekar, J. Lewandowski, D. Marolf, J. Mourão, and T. Thiemann, Quantization of diffeomorphism invariant theories of connections with local degrees of freedom, Jour. Math. Phys., 36 (1995), 6456-6493.

[19] H. Sahlmann, H. Some comments on the representation theory of the algebra underlying loop quantum gravity, gr-qc/0207111.

[20] J. Lewandowski and A. Okolow, Diffeomorphism covariant representations of the holonomy-flux star-algebra, gr-qc/0302059.

[21] H. Sahlmann and T. Thiemann, On the superselection theory of the Weyl algebra for diffeomorphism invariant quantum gauge theories, gr-qc/0302090.

[22] A. Ashtekar, S. Fairhurst and J. Willis, Quantum gravity, shadow states, and quantum mechanics, Class. Quantum Grav., 20 (2003), 1031-1062.

[23] C. Rovelli and L. Smolin, Spin networks and quantum gravity, Phys. Rev., D52 (1995), 5743-5759.

[24] J. C. Baez, Spin networks in non-perturbative quantum gravity, in 'The Interface of Knots and Physics', edited by Kauffman, L. (American Mathematical Society, Providence, 1996) pp. 167-203;

Spin networks in gauge theory, Adv. Math. 117 (1996), 253-272.

[25] M. Bojowald, Isotropic loop quantum cosmology, Class. Quantum Grav., 19 (2002), 2717-2741. 
[26] A. Ashtekar, J. Baez, A. Corichi and K. Krasnov, Quantum geometry and black hole entropy, Phys. Rev. Lett., 80 (1998), 904-907.

A. Ashtekar, J. Baez and K. Krasnov, Quantum geometry of isolated horizons and black hole entropy, Adv. Theo. Math. Phys., 4 (2000), $1-95$.

[27] M. Bojowald, G. Date and K. Vandersloot, Homogeneous loop quantum cosmology: The role of the spin connection, (in preparation).

[28] M. Bojowald, The semiclassical limit of loop quantum cosmology, Class. Quantum Grav., 18 (2002), L109-L116.

[29] M. Gaul and C. Rovelli, A generalized Hamiltonian constraint operator in loop quantum gravity and its simplest Euclidean Matrix Elements, Class. Quantum Grav., 18 (2001), 1593-1624.

[30] C. Rovelli and L. Smolin, Discreteness of area and volume in quantum gravity, Nucl. Phys. B442 (1995), 593-622; Erratum: Nucl. Phys. B456 (1995) 753

S. Frittelli, L. Lehner and C. Rovelli, The complete spectrum of the area from recoupling theory in loop quantum gravity, Class. Quant. Grav. 13 (1996), 2921-2932

[31] Marolf, D. Refined algebraic quantization: Systems with a single constraint, gr-qc/9508015.

[32] T. Thiemann, QSD III: Quantum constraint algebra and physical scalar product in quantum general relativity, Class. Quantum Grav. 15 (1998), $1207-1247$.

[33] M. Bojowald, and H. A. Kastrup, Symmetry reduction for quantized diffeomorphism invariant theories of connections, Class. Quantum Grav., 17 (2000), 3009-3043.

[34] M. Bojowald, Loop quantum cosmology: I. Kinematics, Class. Quantum Grav., 17 (2001), 1489-1508.

[35] A. Ashtekar, J. Lewandowski and H. Sahlmann, Polymer and Fock representations for a Scalar field, Class. Quantum Grav. 20 (2003), L1-11.

[36] R. Gambini and J. Pullin, Discrete quantum gravity: applications to cosmology, gr-qc/0112033.

[37] A. Erdélyi, Asymptotic expansions, Chapter II (Dover, New York, 1956). 